\title{
THE ABSOLUTE LINE QUADRIC AND CAMERA AUTOCALIBRATION
}

\author{
ANTONIO VALDÉS*, JOSÉ IGNACIO RONDA**, AND GUILLERMO GALLEGO**
}

\author{
* Departamento de Geometría y Topología \\ Universidad Complutense de Madrid \\ E-28040 Madrid, Spain \\ Antonio_Valdes@mat.ucm.es
}

\author{
** Grupo de Tratamiento de Imágenes \\ Universidad Politécnica de Madrid \\ E-28040 Madrid, Spain \\ $\{$ jir,ggb\}@gti.ssr.upm.es
}

\begin{abstract}
We introduce a geometrical object providing the same information as the absolute conic: the absolute line quadric (ALQ). After the introduction of the necessary exterior algebra and Grassmannian geometry tools, we analyze the Grassmannian of lines of $\mathbf{P}^{3}$ from both the projective and Euclidean points of view. The exterior algebra setting allows then to introduce the ALQ as a quadric arising very naturally from the dual absolute quadric. We fully characterize the ALQ and provide clean relationships to solve the inverse problem, i.e., recovering the Euclidean structure of space from the ALQ. Finally we show how the ALQ turns out to be particularly suitable to address the Euclidean autocalibration of a set of cameras with square pixels and otherwise varying intrinsic parameters, providing new linear and non-linear algorithms for this problem. We also provide experimental results showing the good performance of the techniques.
\end{abstract}

\section{InTRODUCTION}

Most of the existing methods for the obtainment of 3D reconstructions from sets of uncalibrated views operate in two main steps. The first one provides a projective reconstruction in which $3 \mathrm{D}$ world appears distorted by a space homography. The second one restores the Euclidean structure and provides the camera positions and intrinsic parameters. The first paper to show the possibility of performing this Euclidean upgrading was the seminal paper [13]. No a priori knowledge of the scene was necessary, but only some restrictions in the intrinsic camera parameters, namely their constancy between views. Since then, a wealth of autocalibration techniques considering this or other restrictions appeared. The book [9] is an excellent reference for the topic.

As making an Euclidean upgrading is equivalent to finding the absolute conic [17, p. 254] in the projectively reconstructed scene, all these methods have in common the explicit or implicit consideration of the absolute conic. The absolute conic is a non-degenerated imaginary conic lying on the plane at infinity, with the same equations in any Euclidean coordinate system. One of the main difficulties in finding it is due to the fact that it is a one-dimensional object in a three-dimensional space, i.e., its codimension is two. The finding of objects of codimension one, i.e., given by only one equation, is usually simpler and more stable. The absolute dual quadric (DAQ) [20] came to overcome this problem. This is a rank-three quadric consisting of all the planes which are tangent to the absolute conic, including the plane at infinity itself. It is a codimension-one object of the dual projective space $\mathbf{P}^{3 *}$.

Date: April 5, 2005.

This work has been partly supported by the Comisión Interministerial de Ciencia y Tecnología (CICYT) of the Spanish Government. 
This is not the only codimension-one object equivalent to the absolute conic. There is a standard tool in algebraic geometry called the Chow construction $[8$, p. 268]. This notion has also been considered in [12] to recover algebraic curves from projections. In the case of the absolute conic, the Chow construction consists of all the lines intersecting this conic. Here we study this set of lines using the mapping that assigns to each line the line of its orthogonal directions (quite as the DAQ can be seen as the mapping that assigns to each plane its orthogonal direction). We will term this mapping the absolute line quadric (ALQ).

In a previous work [21] an equivalent object was characterized as a pencil of quadrics in $\mathbf{P}^{5}$. In this paper we pursue this work, showing how the use of exterior algebra provides a deeper geometrical insight resulting in new results and algorithms. More specifically:

1. We provide a new definition of the ALQ that relates it directly with the DAQ.

2. We develop new proofs of known properties of the ALQ which turn out to be very simple as a result of the exterior algebra setting.

3. We introduce a new technique for the direct recovery of the DAQ from the ALQ. 4. We prove a result that allows the direct obtainment of an Euclidean coordinate system from the ALQ (cf. [15]).

5. We provide a new non-linear algorithm for the autocalibration problem combining the use of the ALQ with sequential quadratic programming (SQP) optimization.

A concept which can be proved to be equivalent to the ALQ first appeared in the computer vision literature in [15], in the form of symmetric $6 \times 6$ matrix that characterized the orthogonality of two lines in space expressed in terms of their Plücker coordinates. This work was based in turn in the characterization of zero-skew perspective perspective projection matrices [11][14]. Among other results in [15], we remark the proposal of a procedure to parameterize the space of such square matrices. In this relevant precedent, linear and non-linear autocalibration algorithms were proposed for the case of cameras with rectangular pixels (i.e., with zero-skew projection matrices).

To work with the ALQ, one must first choose a convenient description of the set of lines of space. The choice in this paper is to represent each line by means of a bivector or a bicovector [3][8][16]. This compact geometric treatment is computationally efficient and makes worth the necessary algebraic machinery: exterior algebra, which is receiving an increasing attention within the field of $3 \mathrm{D}$ computer vision, especially in the formulation of Grassmann-Cayley algebra [2][4][6][10]. In [1], geometric algebra is employed for the autocalibration from two cameras with fixed parameters and partial knowledge of the motion. Tensor algebra has been considered as well [19]. Trying to make the paper as self-contained as possible, we provide the reader with the necessary definitions and results in Section 2.

Much of the power of exterior algebra within 3D computer vision lies in its capability to deal with problems involving sums and intersections of linear spaces. One of the contributions of this paper will be a procedure to perform these operations in a general way, thus extending the possibilities of the meet and join operators of the Grassmann-Cayley formulation. This is developed in Section 3.

The study of the ALQ requires a working knowledge of 3D line geometry in the context of exterior algebra that is provided in Section 4. In Section 5 our efforts in developing this mathematical machinery are rewarded with a straightforward expression of the ALQ in terms of the DAQ (see equation (7)). We then see how the ALQ encodes the Euclidean structure of space and how we can cleanly recover the DAQ from the knowledge of the ALQ. This is one of the main contributions of this paper. 
In [21] a linear algorithm was theoretically proposed to recover the Euclidean structure of space from of a set of cameras with known pixel shape and varying parameters using the calibration pencil. In Section 6 it is shown how the developed exterior algebra techniques of Section 5 allow for an improvement of this algorithm. We also provide a more costly non-linear algorithm showing a significantly better performance. Experimental results for these algorithms are provided.

\section{INTRODUCTION TO TENSOR AND EXTERIOR ALGEBRA}

In this section we summarize the necessary algebraic machinery for the paper. A general reference for this topic is [3].

We start with some basic notation. The field of complex numbers will be denoted by C. If $V$ is a complex vector space, we will denote by $V^{*}$ the dual space of $V$, i.e., the vector space of linear mappings $\alpha: V \rightarrow \mathbf{C}$. If $\left\{v_{0}, \ldots, v_{n}\right\}$ is a basis of $V$, we denote by $\left\{v_{0}^{*}, \ldots, v_{n}^{*}\right\}$ its dual basis of $V^{*}$, defined by $v_{i}^{*}\left(v_{j}\right)=\delta_{i j}$ where $\delta_{i j}$ stands for Kronecker's delta. The projective space associated to $V$ will be denoted by $P(V)$. In the case that $V=\mathbf{C}^{n}$, we will write $\mathbf{P}^{n}=P\left(\mathbf{C}^{n}\right)$. Equality up to a non-zero scale factor will be denoted by $\sim$.

2.1. Tensor product. Let $V$ and $W$ be complex vector spaces. The tensor product $V \otimes W$ is a complex vector space generated by the linear combinations of the formal expressions $v \otimes w$ for $v \in V$ and $w \in W$, where the following identities are imposed to hold true:

$$
\begin{aligned}
\left(\lambda_{1} v_{1}+\lambda_{2} v_{2}\right) \otimes w & =\lambda_{1} v_{1} \otimes w+\lambda_{2} v_{2} \otimes w \\
v \otimes\left(\lambda_{1} w_{1}+\lambda_{2} w_{2}\right) & =\lambda_{1} v \otimes w_{1}+\lambda_{2} v \otimes w_{2}
\end{aligned}
$$

for any $\lambda_{1}, \lambda_{2} \in \mathbf{C}, v, v_{1}, v_{2} \in V$ and any $w, w_{1}, w_{2} \in W$. An alternative more formal definition of tensor product can be found in [3].

Let $\left\{v_{0}, \ldots, v_{n}\right\}$ and $\left\{w_{0}, \ldots, w_{m}\right\}$ be bases of $V$ and $W$, respectively (we use indices from 0 in order that the last index coincides with the dimension of the associated projective space). The elements of the form $v_{i} \otimes w_{j}$, constitute a basis of $V \otimes W$, so any element $a \in V \otimes W$ can be written as $a=\sum_{i, j} a_{i j} v_{i} \otimes w_{j}$ for certain coefficients $a_{i j} \in \mathbf{C}$. Consequently the dimension of $V \otimes W$ is the product of the dimensions of the factors. This construction generalizes to the tensor product of any number of complex vector spaces. In particular we will denote by $\otimes^{k} V$ the $k$-fold tensor product $V \otimes \cdots \otimes V$.

2.2. Contractions. Given $b=u_{1} \otimes \cdots \otimes u_{k} \in \otimes^{k} V$ and $\beta=\alpha_{1} \otimes \cdots \otimes \alpha_{l} \in$ $\bigotimes^{l} V^{*}$, and a pair of indexes $1 \leq i \leq k, 1 \leq j \leq l$, we define the contraction $C_{j}^{i}(a \otimes \beta) \in \bigotimes^{k-1} V \otimes \bigotimes^{l-1} V^{*}$ by the rule

$$
C_{j}^{i}(a \otimes \beta)=\alpha_{j}\left(u_{i}\right) u_{1} \otimes \cdots \otimes \hat{u}_{i} \otimes \cdots \otimes u_{k} \otimes \alpha_{1} \otimes \cdots \otimes \hat{\alpha}_{j} \otimes \cdots \otimes \alpha_{l},
$$

where $\hat{u}_{i}$ and $\hat{\alpha}_{j}$ stand for eliminated elements. Extended by linearity, the operation $C_{j}^{i}$ defines a linear mapping

$$
C_{j}^{i}: \bigotimes^{k} V \otimes \bigotimes^{l} V^{\star} \rightarrow \bigotimes^{k-1} V \otimes \bigotimes^{l-1} V^{\star}
$$

2.3. Interpretation of $V \otimes V^{*}$. The tensor product $V \otimes V^{*}$ can be identified either with the space $\operatorname{End}(V)$ of endomorphisms of $V$ (i.e., the set of linear mappings of $V$ on itself) or with $\operatorname{End}\left(V^{*}\right)$. The identification is very natural, for given $\alpha=V \otimes V^{*}$ we can define a mapping $V \rightarrow V$ sending $V \ni w \mapsto C_{1}^{1}(w \otimes \alpha) \in V$. Analogously, the endomorphism of $V^{*}$ corresponding to $\alpha$ is given by $V^{*} \ni \gamma \mapsto C_{1}^{2}(\alpha \otimes \gamma) \in V^{*}$.

To see this in terms of coordinates, consider a basis $\left\{v_{0}, \ldots, v_{n}\right\}$ of $V$ and its dual basis $\left\{v_{0}^{*}, \ldots, v_{n}^{*}\right\}$ of $V^{*}$. We can write $\alpha=\sum_{i, j} \alpha_{i j} v_{i} \otimes v_{j}^{*}$ and $w=\sum_{i} \lambda_{i} v_{i}$, 
we have that $C_{1}^{1}(w \otimes \alpha)=\sum_{i}\left(\sum_{j} \alpha_{i j} \lambda_{j}\right) v_{i}$. So the matrix of the endomorphism is given by $\left(\alpha_{i j}\right)$. Analogously, the endomorphism of $V^{*}$ is given by the transposed matrix $\left(\alpha_{i j}\right)^{t}$. From this it easily follows that decomposable tensors of $V \otimes V^{*}$, i.e., elements of the form $a \otimes \beta \in V \otimes V^{*}$, are characterized by having matrices of rank one.

2.4. Exterior product. The subspace of $V \otimes V$ spanned by the elements of the form $v \otimes w-w \otimes v, v, w \in V$, is called the exterior product space $V \wedge V=\wedge^{2} V$. We will denote by $\wedge$ the exterior or wedge product $v \wedge w=\frac{1}{2}(v \otimes w-w \otimes v)$. Alternatively, $V \wedge V$ can be seen as the vector space generated by linear combinations of formal expressions $v \wedge w$, with $v, w \in V$ where the following identities hold:

$$
\begin{aligned}
\left(\lambda_{1} v_{1}+\lambda_{2} v_{2}\right) \wedge w & =\lambda_{1} v_{1} \wedge w+\lambda_{2} v_{2} \wedge w \\
v \wedge\left(\lambda_{1} w_{1}+\lambda_{2} w_{2}\right) & =\lambda_{1} v \wedge w_{1}+\lambda_{2} v \wedge w_{2} \\
v \wedge w & =-w \wedge v .
\end{aligned}
$$

Elements of $\bigwedge^{2} V$ will be called bivectors. Analogously, elements of $\bigwedge^{2} V^{*}$ will be called bicovectors. Given a basis $\left\{v_{0}, \ldots, v_{n}\right\}$ of $V$, the $\left(\begin{array}{c}n+1 \\ 2\end{array}\right)$ elements of the form $v_{i} \wedge v_{j}, 0 \leq i<j \leq n$ constitute a basis of $V \wedge V$.

Analogously, we can define the exterior product $\bigwedge^{k} V \subset \bigotimes^{k} V$ as the subspace generated by the expressions of the form

$$
u_{1} \wedge \cdots \wedge u_{k}=\frac{1}{r !} \sum_{\sigma}(-1)^{|\sigma|} u_{\sigma_{1}} \otimes \cdots \otimes u_{\sigma_{k}}
$$

where $\sigma$ runs over the set of permutations of $\{1, \ldots, k\}$ and $|\sigma|$ stands for its signature. We will also denote by $|a|$ the degree of $a$, i.e., $|a|=k$ if $a \in \bigwedge^{k} V$. The following relation is easy to prove:

$$
u_{1} \wedge \cdots \wedge u_{k}=(-1)^{|\sigma|} u_{\sigma_{1}} \wedge \cdots \wedge u_{\sigma_{k}} .
$$

A basis of $\bigwedge^{k} V$ is given by the $\left(\begin{array}{c}n+1 \\ k\end{array}\right)$ elements of the form $v_{i_{1}} \wedge \cdots \wedge v_{i_{k}}, 0 \leq i_{1}<$ $\cdots<i_{k} \leq n$.

In the particular case $k=n+1$ it turns out that $\bigwedge^{n+1} V$ is one-dimensional, so any non-zero element of $\bigwedge^{n+1} V$ defines a basis, which permits to identify $\bigwedge^{n+1} V$ with $\mathbf{C}$. Such an element will be called a volume form. The other extreme case corresponds to $k=0$. By definition $\bigotimes^{0} V=\bigwedge^{0} V=\mathbf{C}$.

Given $a \in \bigwedge^{k} V$ and $a^{\prime} \in \bigwedge^{l} V$, we define $a \wedge a^{\prime} \in \bigwedge^{k+l} V$ as follows. If $a$ and $a^{\prime}$ are decomposable, i.e., $a=u_{1} \wedge \cdots \wedge u_{k}$ and $a^{\prime}=u_{1}^{\prime} \wedge \cdots \wedge u_{l}^{\prime}$, then $a \wedge a^{\prime}=u_{1} \wedge \cdots \wedge u_{k} \wedge u_{1}^{\prime} \wedge \cdots \wedge u_{l}^{\prime}$, and this extends linearly to linear combinations of decomposable elements. From this it readily follows that for any $a \in \wedge^{k} V$ and $a^{\prime} \in \bigwedge^{l} V$ we have

$$
a \wedge a^{\prime}=(-1)^{|a|\left|a^{\prime}\right|} a^{\prime} \wedge a .
$$

2.5. Contractions in exterior products. Given $r \leq p, q$, the iterated composition $C_{[r]}:=\left(C_{1}^{1}\right)^{r}$ restricts to an operator $C_{[r]}: \bigwedge^{p} V \otimes \bigwedge^{q} V^{*} \rightarrow \bigwedge^{p-r} V \otimes \bigwedge^{q-r} V^{*}$. In particular, using the expansion (1), it is not difficult to derive the formula

$$
\begin{aligned}
& C_{[1]}\left(u_{1} \wedge \ldots \wedge u_{r} \otimes \alpha_{1} \wedge \ldots \wedge \alpha_{s}\right)= \\
& \frac{1}{r s} \sum_{i, j}(-1)^{i+j} \alpha_{j}\left(u_{i}\right)\left(u_{1} \wedge \ldots \wedge \hat{u}_{i} \wedge \ldots \wedge u_{r}\right) \otimes\left(\alpha_{1} \wedge \ldots \wedge \hat{\alpha}_{j} \wedge \ldots \wedge \alpha_{s}\right),
\end{aligned}
$$

where the hat ^ denotes the suppression of the element. 
Let us illustrate this with some examples. First consider $u \in V$ and $\alpha \wedge \beta \in$ $\bigwedge^{2} V^{*}$. The contraction $C_{[1]}(u \otimes(\alpha \wedge \beta))$ is given by

$$
C_{[1]}(u \otimes(\alpha \wedge \beta))=\frac{1}{2}(\alpha(u) \beta-\beta(u) \alpha) .
$$

As a second example, we consider $u \wedge v \in \bigwedge^{2} V$ and $\alpha \wedge \beta \in \bigwedge^{2} V^{*}$. A direct computation produces

$$
\begin{aligned}
& C_{[1]}((u \wedge v) \otimes(\alpha \wedge \beta))=\frac{1}{4}(\alpha(u) v \otimes \beta-\beta(u) v \otimes \alpha-\alpha(v) u \otimes \beta+\beta(v) u \otimes \alpha), \\
& C_{[2]}((u \wedge v) \otimes(\alpha \wedge \beta))=\frac{1}{2}(\alpha(u) \beta(v)-\beta(u) \alpha(v)) .
\end{aligned}
$$

2.6. Dual of $\bigwedge^{p} V$. We have the canonical identification

$$
\left(\bigwedge^{p} V\right)^{*}=\bigwedge^{p} V^{*}
$$

in which an element $\alpha_{1} \wedge \cdots \wedge \alpha_{p} \in \wedge^{p} V^{*}$ is interpreted as a linear mapping $\bigwedge^{p} V \rightarrow \mathbf{C}$ as follows:

$$
\begin{aligned}
& \left(\alpha_{1} \wedge \cdots \wedge \alpha_{p}\right)\left(u_{1} \wedge \cdots \wedge u_{p}\right)=C_{[p]}\left(\left(u_{1} \wedge \cdots \wedge u_{p}\right) \otimes\left(\alpha_{1} \wedge \cdots \wedge \alpha_{p}\right)\right) \\
& =\frac{1}{p !} \sum_{\sigma}(-1)^{|\sigma|} \alpha_{1}\left(u_{\sigma_{1}}\right) \cdots \alpha_{p}\left(u_{\sigma_{p}}\right)
\end{aligned}
$$

where $\sigma$ runs over all the permutations of $\{1, \ldots, p\}$. From this it follows that $\left\{p ! v_{i_{1}}^{*} \wedge \cdots \wedge v_{i_{p}}^{*}: i_{1}<\cdots<i_{p}\right\}$ is the dual basis of $\left\{v_{i_{1}} \wedge \cdots \wedge v_{i_{p}}: i_{1}<\cdots<i_{p}\right\}$.

\section{Exterior Algebra AND Linear subspaces of $\mathbf{P}^{n}$}

3.1. Representations of linear subspaces as decomposable multivectors and multicovectors. The Grassmannian $G(k+1, V)$ is defined as the set of all $(k+1)$-dimensional subspaces of $V$. If $V=\mathbf{C}^{n+1}$, it can be identified with the $k$-dimensional subspaces of $\mathbf{P}^{n}$. When we have in mind this second interpretation, we will employ the notation $G\left(k+1, \mathbf{C}^{n+1}\right)=\mathbb{G}(k, n)$. Let $W \subset V$ be a linear subspace. Taking any basis of $W,\left\{w_{0}, \ldots, w_{k}\right\}$, we can build the multivector $w_{0} \wedge \cdots \wedge w_{k} \in \wedge^{k+1} V$, which is uniquely defined by $W$ up to a non-zero scalar factor: in fact, if $\left\{w_{0}^{\prime}, \ldots, w_{k}^{\prime}\right\}$ is another basis of $W$, we have that $w_{0}^{\prime} \wedge \cdots \wedge w_{k}^{\prime}=$ $\operatorname{det}(M) w_{0} \wedge \cdots \wedge w_{k}$ where $M$ is the matrix of the change of basis. Therefore the class $\left[w_{0} \wedge \cdots \wedge w_{k}\right] \in P\left(\wedge^{k+1} V\right)$ is uniquely determined by $W$. Conversely, given a decomposable $(k+1)$-vector $a=u_{0} \wedge \cdots \wedge u_{k} \neq 0$, we can recover the associated vector space as the set of those vectors $u$ such that $u \wedge a=0$. This permits to identify $G(k+1, V)$ with the set of decomposable elements of $P\left(\bigwedge^{k+1} V\right)$. This is the generator-based representation of the vector subspace.

Given a subspace $W \subset V$ of dimension $k$, we define its annihilator $W^{\circ} \subset V^{*}$ as the set of those covectors $\alpha \in V^{*}$ vanishing on $W . W^{\circ}$ is a vector subspace of $V^{*}$ of dimension $n-k$. Analogously, given a subspace $\Lambda \subset V^{*}$, we can define its annihilator $\Lambda^{\circ}$ as those vectors $v \in V$ such that $\alpha(v)=0$ for any $\alpha \in \Lambda$. This defines a bijection between $G(k+1, V)$ and $G\left(n-k, V^{*}\right)$. Consequently, we can also represent the elements of the Grassmannian $G(k+1, V)$ using decomposable $(n-k)$-covectors of $\bigwedge^{n-k} V^{*}$. This is the equation-based representation of the subspace. We will see later that, in fact, there exists an isomorphism, defined up to a scalar factor, between $\bigwedge^{k+1} V$ and $\bigwedge^{n-k} V^{*}$ corresponding to this bijection.

Special mention deserve the cases of multivectors and multicovectors of order 0 and $n+1$. The only element of $P\left(\bigwedge^{0} V\right)=P(\mathbf{C})$ is the class [1], and the only element of $P\left(\bigwedge^{n+1} V^{*}\right)$ is the class $\left[v_{0} \wedge \cdots \wedge v_{n}\right]$. Both are interpreted as representatives of the only element of $G(0, V)$, namely the 0 -vector space which projectively represents 
the empty set. Analogously, the only elements of $P\left(\bigwedge^{n+1} V\right)$ and of $P\left(\bigwedge^{0} V^{*}\right)$ represent the total space.

3.1.1. Example: $A$ line of $\mathbf{P}^{2}$. The points $\left(x_{0}, y_{0}, z_{0}\right)$ and $\left(x_{1}, y_{1}, z_{1}\right)$ are represented by vectors $a=x_{0} v_{0}+y_{0} v_{1}+z_{0} v_{2}$ and $b=x_{1} v_{0}+y_{1} v_{1}+z_{1} v_{2}$. The line $a b$ is represented by the bivector $a \wedge b=\left(x_{0} y_{1}-x_{1} y_{0}\right) v_{0} \wedge v_{1}+\left(y_{0} z_{1}-y_{1} z_{0}\right) v_{1} \wedge v_{2}+$ $\left(z_{0} x_{1}-z_{1} x_{0}\right) v_{2} \wedge v_{0}$. The points of the line are given by vectors $c=x v_{0}+y v_{1}+z v_{2}$ such that $(a \wedge b) \wedge c=D v_{0} \wedge v_{1} \wedge v_{2}=0$, where $D$ is the determinant of the matrix defined by the coefficients of the vectors $a, b$ and $c$, so we recover the equation of the line $\left(x_{0} y_{1}-x_{1} y_{0}\right) x-\left(y_{0} z_{1}-y_{1} z_{0}\right) y+\left(z_{0} x_{1}-z_{1} x_{0}\right) z=0$. The covector $\alpha=\left(x_{0} y_{1}-x_{1} y_{0}\right) v_{0}^{*}-\left(y_{0} z_{1}-y_{1} z_{0}\right) v_{1}^{*}+\left(z_{0} x_{1}-z_{1} x_{0}\right) v_{2}^{*}$ is the equation-based representation of the line.

3.2. Switching between generators and equations of a linear subspace. Let us consider a volume form $\mathfrak{V}=v_{0} \wedge \cdots \wedge v_{n} \in \wedge^{n+1} V$ which permits to identify $\wedge^{n+1} V$ with $\mathbf{C}$.

The wedge product

$$
\wedge: \bigwedge^{p} V \times \bigwedge^{n+1-p} V \rightarrow \mathbf{C} \equiv \bigwedge^{n+1} V
$$

can be seen as a bilinear mapping which assigns to $\alpha \in \bigwedge^{p} V$ and $\beta \in \bigwedge^{n-p+1} V$ the scalar $\langle\alpha, \beta\rangle$ characterized by

$$
\alpha \wedge \beta=\langle\alpha, \beta\rangle \mathfrak{V} .
$$

This mapping permits to identify $\bigwedge^{p} V$ with $\bigwedge^{n+1-p} V^{*}$ by assigning to $\alpha \in \bigwedge^{p} V$ the element $A \alpha \in \Lambda^{n+1-p} V^{*}$ defined by

$$
\begin{aligned}
A \alpha: \bigwedge^{n+1-p} V & \rightarrow \mathbf{C} \\
\beta & \mapsto A \alpha(\beta)=\langle\alpha, \beta\rangle .
\end{aligned}
$$

In other words, the defining relation of $A: \bigwedge^{p} V \rightarrow \bigwedge^{n+1-p} V^{*}$ is

$$
\alpha \wedge \beta=(A \alpha)(\beta) \mathfrak{V} .
$$

Let us see how this mapping is expressed in terms of the basis of $\bigwedge^{p} V$ associated to our basis of $V$. We introduce the following notation: If $I=\left(i_{1}, \ldots, i_{k}\right)$ is an increasing sequence of different indexes, we define $v_{I}=v_{i_{1}} \wedge \cdots \wedge v_{i_{k}}$. We also define $I^{\prime}$ as the increasing complement of $I$ in $\{0, \ldots, n\}$. Then

$$
A v_{I}=(-1)^{\left|I, I^{\prime}\right|} v_{I^{\prime}}^{*}
$$

where $(-1)^{\left|I, I^{\prime}\right|}$ is the signature of the permutation $\left(I, I^{\prime}\right)$ of $\{1, \ldots, n\}$. The proof of this fact can be found in the Appendix.

It is worth to remark that $A$ is defined up to a scalar multiple, since it depends on the choice of the volume form. Therefore, it leads to an intrinsic isomorphism between the associated projective spaces, $P\left(\bigwedge^{k+1} V\right)$ and $P\left(\bigwedge^{n-k} V^{*}\right)$. Note that if $a \in \bigwedge^{k+1} V$ is a decomposable $(k+1)$-vector representing a linear subspace $W$, then $A a \in \bigwedge^{n-k} V^{*}$ is the multicovector representing its annihilator $W^{\circ}$. To see this, it is enough to use an adapted basis $\left\{v_{0}, \ldots, v_{n}\right\}$ such that $a \sim v_{0} \wedge \cdots \wedge v_{k}$ so that $A a=v_{k+1}^{*} \wedge \cdots \wedge v_{n}^{*}$. Now $W$ is given by the equations $v_{k+1}^{*}(v)=\cdots=v_{n}^{*}(v)=0$. 
3.2.1. Example: Changing representations in $\mathbf{P}^{3}$. Particularly important for our purposes is the case of the three dimensional projective space, so let us suppose $\operatorname{dim} V=4$. Then, the operator $A$ associated to the volume form $v_{0} \wedge v_{1} \wedge v_{2} \wedge v_{3}$ is given by the following table:

$$
\begin{array}{lll}
1 \mapsto v_{0}^{*} \wedge v_{1}^{*} \wedge v_{2}^{*} \wedge v_{3}^{*} & v_{0} \wedge v_{1} \mapsto v_{2}^{*} \wedge v_{3}^{*} & v_{0} \wedge v_{1} \wedge v_{2} \mapsto v_{3}^{*} \\
& v_{0} \wedge v_{2} \mapsto-v_{1}^{*} \wedge v_{3}^{*} & v_{0} \wedge v_{1} \wedge v_{3} \mapsto-v_{2}^{*} \\
v_{0} \mapsto v_{1}^{*} \wedge v_{2}^{*} \wedge v_{3}^{*} & v_{0} \wedge v_{3} \mapsto v_{1}^{*} \wedge v_{2}^{*} & v_{0} \wedge v_{2} \wedge v_{3} \mapsto v_{1}^{*} \\
v_{1} \mapsto-v_{0}^{*} \wedge v_{2}^{*} \wedge v_{3}^{*} & v_{1} \wedge v_{2} \mapsto v_{0}^{*} \wedge v_{3}^{*} & v_{1} \wedge v_{2} \wedge v_{3} \mapsto-v_{0}^{*} \\
v_{2} \mapsto-v_{0}^{*} \wedge v_{1}^{*} \wedge v_{3}^{*} & v_{1} \wedge v_{3} \mapsto-v_{0}^{*} \wedge v_{2}^{*} & \\
v_{3} \mapsto v_{0}^{*} \wedge v_{1}^{*} \wedge v_{2}^{*} & v_{2} \wedge v_{3} \mapsto v_{0}^{*} \wedge v_{1}^{*} & v_{0} \wedge v_{1} \wedge v_{2} \wedge v_{3} \mapsto 1
\end{array}
$$

3.3. Sum and intersection of linear subspaces. The sum and intersection of linear subspaces are fundamental operations in Computer Vision. The most extended setting to perform these operations is given by the Grassmann-Cayley algebra with its operators meet and join [2] [6]. This provides a nice double-algebra structure which facilitates both geometric intuition and computations. However, a drawback of this approach is that if $a$ and $b$ are representatives of subspaces $A$ and $B$, the meet operator gives a representative of $A \cap B$ only if $A+B$ spans the whole space. Analogously, the join operator fails to compute the sum of subspaces unless their intersection is the null vector space. So, the meet operator fails when we try to check whether a given point lies or not on a line in $\mathbf{P}^{3}$ or to compute the intersection point of two coplanar lines, and the join operator fails to compute, for example, the plane of two intersecting lines. Definitions of the meet and join operators and their relationship with exterior algebra are given in the Appendix.

These considerations lead us to the necessity of providing a general procedure to compute the intersection and sum of two arbitrary linear subspaces. The result is given by the following Theorem.

Theorem 3.1. Let $U$ and $U^{\prime}$ be two linear subspaces of $V$ and let $a \in \bigwedge^{p} V$ and $a^{\prime} \in \bigwedge^{p^{\prime}} V$ be representatives of them. Let $k$ be the highest integer such that $k \leq \min \left\{p, n+1-p^{\prime}\right\}$ and the contraction $C_{[k]}\left(a \otimes A a^{\prime}\right)$ (which coincides with $\left.\pm \bar{C}_{[k]}\left(a^{\prime} \otimes A a\right)\right)$ does not vanish. Then $C_{[k]}\left(a \otimes A a^{\prime}\right)=b \otimes \beta$ where $b$ is a multivector representative of $U \cap U^{\prime}$ and $\beta$ is a multicovector representative of $U+U^{\prime}$.

Proof. Let us take a basis of $V,\left\{v_{i}\right\}$ adapted to $U$ and $U^{\prime}$ in the sense that $U$ is represented by $v_{I} \wedge v_{J}$ and $U^{\prime}$ is represented by $v_{J} \wedge v_{K}$, being the multi-indexes $I, J$ and $K$ pairwise disjoint. Note that $U \cap U^{\prime}$ is represented by $v_{J}$ and $U+U^{\prime}$ is represented by $v_{I} \wedge v_{J} \wedge v_{K}$. Then $A\left(v_{J} \wedge v_{K}\right)= \pm v_{I}^{*} \wedge v_{L}^{*}$, where $L$ is the multiindex representing the remaining indexes not in $I, J$ and $K$. Then $k$ is the length of $I$, and $C_{[k]}\left(v_{I} \wedge v_{J} \otimes A\left(v_{J} \wedge v_{K}\right)\right)= \pm v_{J} \otimes v_{L}^{*}$, so $b=v_{J}$ represents $U \cap U^{\prime}$ and $v_{L}^{*}= \pm A\left(v_{I} \wedge v_{J} \wedge v_{K}\right)$ represents the equations of the sum $U+U^{\prime}$. An analogous computation proves that $C_{[k]}\left(a \otimes A a^{\prime}\right)= \pm C_{[k]}\left(a^{\prime} \otimes A a\right)$.

This Theorem has an immediate application to the case of linear projective subspaces. Let us illustrate the result with some examples.

3.3.1. A point on a line of $\mathbf{P}^{3}$. A line in $\mathbf{P}^{3}$ through the points represented by vectors $a=a_{0} v_{0}+a_{1} v_{1}+a_{2} v_{2}+a_{3} v_{3}$ and $b=b_{0} v_{0}+b_{1} v_{1}+b_{2} v_{2}+b_{3} v_{3}$ is represented by the bivector

$$
\begin{aligned}
a \wedge b= & \left(a_{0} b_{1}-b_{0} a_{1}\right) v_{0} \wedge v_{1}+\left(a_{0} b_{2}-b_{0} a_{2}\right) v_{0} \wedge v_{2}+\left(a_{0} b_{3}-b_{0} a_{3}\right) v_{0} \wedge v_{3}+ \\
& \left(a_{1} b_{2}-b_{1} a_{2}\right) v_{1} \wedge v_{2}+\left(a_{2} b_{3}-b_{2} a_{3}\right) v_{2} \wedge v_{3}+\left(a_{3} b_{0}-b_{3} a_{0}\right) v_{3} \wedge v_{1}
\end{aligned}
$$


TABLE 1. Representation of linear subspaces of $\mathbf{P}^{3}$.

\begin{tabular}{|c|c|c|}
\hline$\emptyset$ & $P\left(\bigwedge^{0} V\right)=[1]$ & $P\left(\bigwedge^{4} V^{*}\right)=\left[v_{0}^{*} \wedge v_{1}^{*} \wedge v_{2}^{*} \wedge v_{3}^{*}\right]$ \\
\hline Points & $P\left(\bigwedge^{1} V\right)=\mathbf{P}^{3}$ & $P\left(\bigwedge^{3} V^{*}\right)$ \\
\hline Lines & $\mathbb{G}(1,3) \subset P\left(\bigwedge^{2} V\right)$ & $\mathbb{G}(1,3) \subset P\left(\bigwedge^{2} V^{*}\right)$ \\
\hline Planes & $P\left(\bigwedge^{3} V\right)$ & $P\left(\bigwedge^{1} V^{*}\right)=\mathbf{P}^{3 *}$ \\
\hline $\mathbf{P}^{3}$ & $P\left(\bigwedge^{4} V\right)=\left[v_{0} \wedge v_{1} \wedge v_{2} \wedge v_{3}\right]$ & $P\left(\bigwedge^{0} V^{*}\right)=[1]$ \\
\hline
\end{tabular}

The equation-based representation is given by

$$
\begin{aligned}
A(a \wedge b)= & \left(a_{0} b_{1}-b_{0} a_{1}\right) v_{2}^{*} \wedge v_{3}^{*}-\left(a_{0} b_{2}-b_{0} a_{2}\right) v_{1}^{*} \wedge v_{3}^{*}+\left(a_{0} b_{3}-b_{0} a_{3}\right) v_{1}^{*} \wedge v_{2}^{*}+ \\
& \left(a_{1} b_{2}-b_{1} a_{2}\right) v_{0}^{*} \wedge v_{3}^{*}+\left(a_{2} b_{3}-b_{2} a_{3}\right) v_{0}^{*} \wedge v_{1}^{*}+\left(a_{3} b_{0}-b_{3} a_{0}\right) v_{0}^{*} \wedge v_{2}^{*} .
\end{aligned}
$$

A vector $x=x_{0} v_{0}+x_{1} v_{1}+x_{2} v_{2}+x_{3} v_{3}$ belong to the line $a b$ iff $C_{[1]}(x \otimes A(a \wedge b))=0$ or, equivalently, iff $a \wedge b \wedge x=0$. Any of these methods leads us to the equations

$$
\begin{array}{r}
\left(-a_{3} b_{4}+a_{4} b_{3}\right) x_{2}+\left(a_{2} b_{4}-a_{4} b_{2}\right) x_{3}+\left(-a_{2} b_{3}+a_{3} b_{2}\right) x_{4}=0 \\
\left(a_{3} b_{4}-a_{4} b_{3}\right) x_{1}+\left(-a_{1} b_{4}+a_{4} b_{1}\right) x_{3}+\left(a_{1} b_{3}-a_{3} b_{1}\right) x_{4}=0 \\
\left(-a_{2} b_{4}+a_{4} b_{2}\right) x_{1}+\left(a_{1} b_{4}-a_{4} b_{1}\right) x_{2}+\left(-a_{1} b_{2}+a_{2} b_{1}\right) x_{4}=0 \\
\left(a_{2} b_{3}-a_{3} b_{2}\right) x_{1}+\left(-a_{1} b_{3}+a_{3} b_{1}\right) x_{2}+\left(a_{1} b_{2}-a_{2} b_{1}\right) x_{3}=0 .
\end{array}
$$

Note that these are the equations of the four planes passing through the points $a$ and $b$ and through the vertices of the coordinate tetrahedron. Generically, any two of them define the line $a b$.

For the reader's convenience we have summarized in Table 1 the correspondence between linear subspaces and multivectors or multicovectors of $\mathbf{P}^{3}$.

3.3.2. Relative positions of two lines in $\mathbf{P}^{3}$. Let $r$ and $r^{\prime}$ be two lines given respectively by bivectors $a=u \wedge v$ and $a^{\prime}=u^{\prime} \wedge v^{\prime}$. There are three different possibilities:

(1) $C_{[1]}\left(a \otimes A a^{\prime}\right)=0$. Since $C_{[0]}$ is the identity mapping, $C_{[0]}\left(a \otimes A a^{\prime}\right)=a \otimes A a^{\prime}$, therefore both lines are coincident.

(2) If $u \otimes \alpha=C_{[1]}\left(a \otimes A a^{\prime}\right) \neq 0$ and $C_{[2]}\left(a \otimes A a^{\prime}\right)=0$, the both lines intersect at the point represented by $u$ and are contained in the plane given by the covector $\alpha$.

(3) If $C_{[1]}\left(a \otimes A a^{\prime}\right) \neq 0$ and $C_{[2]}\left(a \otimes A a^{\prime}\right) \neq 0$ then necessarily $C_{[2]}\left(a \otimes A a^{\prime}\right) \sim$ $1 \otimes 1$, so the intersection is empty and the sum is the total space.

3.3.3. Two intersecting lines in $\mathbf{P}^{3}$. We consider the lines in $\mathbf{P}^{3}$ of equations $r_{1}$ : $x_{1}=x_{2}+x_{3}=0$ and $r_{2}: x_{0}+x_{2}=x_{0}-x_{3}=0$. First, we find representatives of these lines in $\bigwedge^{2} V^{*}$, which is immediate from their equations: $\alpha_{1}=v_{1}^{*} \wedge\left(v_{2}^{*}+v_{3}^{*}\right)$ and $\alpha_{2}=\left(v_{0}^{*}+v_{2}^{*}\right) \wedge\left(v_{0}^{*}-v_{3}^{*}\right)$. Then we apply $A^{-1}$ to one of them, say $\alpha_{1}$, using for instance the table in 3.2.1, obtaining $a=A^{-1}\left(\alpha_{1}\right)=v_{0} \wedge v_{3}-v_{0} \wedge v_{2}$. Then we compute

$$
\begin{aligned}
C_{[1]}\left(a \otimes \alpha_{2}\right) & =\frac{1}{4}\left(-v_{3} \otimes v_{3}^{*}-v_{3} \otimes v_{2}^{*}-v_{0} \otimes v_{2}^{*}+v_{2} \otimes v_{3}^{*}+v_{2} \otimes v_{2}^{*}-v_{0} \otimes v_{3}^{*}\right) \\
& =-\frac{1}{4}\left(v_{0}-v_{2}+v_{3}\right) \otimes\left(v_{2}^{*}+v_{3}^{*}\right),
\end{aligned}
$$

which is non-zero. We also have that $C_{[2]}\left(a_{1} \otimes \alpha_{2}\right)=0$. Consequently the intersection of $r_{1}$ and $r_{2}$ is represented by the vector $v_{0}-v_{2}+v_{3}$, which corresponds to the point $(1,0,-1,1)$, and the plane containing both lines has equation $x_{2}+x_{3}=0$.

Note that once computed $C_{[1]}\left(a \otimes \alpha_{2}\right)$, to factorize the result in the form $b \otimes \beta$ is just a matter of collecting the factors that multiply each basis element $v_{I}^{*}$, which can be done contracting the result with $\frac{1}{p !} v_{I}$, where $p$ is the order of the multicovector. 
3.3.4. Intersection of a line and a plane in $\mathbf{P}^{3}$. To compute the intersection of the line $r_{1}: x_{1}=x_{2}+x_{3}=0$ and the plane $x_{0}-x_{3}=0$ we take the representative of the line $a=v_{0} \wedge v_{3}-v_{0} \wedge v_{2} \in \wedge^{2} V$ computed above and the representative of the plane $\beta=v_{0}^{*}-v_{3}^{*}$. The contraction

$$
C_{[1]}(a \otimes \beta)=-\frac{1}{2}\left(-v_{3}+v_{4}\right)
$$

represents the intersection point $(0,0,-1,1)$ and since $-\frac{1}{2}\left(-v_{3}+v_{4}\right)=-\frac{1}{2}\left(-v_{3}+\right.$ $\left.v_{4}\right) \otimes 1$ we obtain that their sum is represented by $1 \in \bigwedge^{0} V^{*}=\mathbf{C}$, i.e., it is the total space $\mathbf{P}^{3}$.

3.3.5. Two non-intersecting lines in $\mathbf{P}^{3}$. We now consider the lines in $\mathbf{P}^{3}$ of equations $r_{1}: x_{1}=x_{2}+x_{3}=0$ and $r_{2}: x_{0}+x_{2}=x_{1}-x_{3}=0$. Analogously to the previous examples, we take the previously computed representative $a$ of $r_{1}$ and $\beta=\left(v_{0}^{*}+v_{2}^{*}\right) \wedge\left(v_{1}^{*}-v_{3}^{*}\right)$ of $r_{2}$. We compute

$$
\begin{aligned}
C_{[1]}(a \otimes \beta) & =\frac{1}{4}\left(-v_{0} \otimes v_{0}^{*}+v_{0} \otimes v_{1}^{*}-v_{0} \otimes v_{2}^{*}-v_{0} \otimes v_{3}^{*}\right. \\
& \left.-v_{2} \otimes v_{1}^{*}+v_{2} \otimes v_{3}^{*}+v_{3} \otimes v_{1}^{*}-v_{3} \otimes v_{3}^{*}\right) \\
C_{[2]}(a \otimes \beta) & =-\frac{1}{2}=-\frac{1}{2} 1 \otimes 1,
\end{aligned}
$$

so we conclude that the intersection is empty, since it is represented by $1 \in \bigwedge^{0} V$ and their sum is the total space $\mathbf{P}^{3}$, since it is represented by $1 \in \bigwedge^{0} V^{*}$.

\section{Geometry of Lines in $\mathbf{P}^{3}$}

4.1. The Klein quadric. Only decomposable bivectors, i.e., those of the form $a=u \wedge v$ represent lines. Clearly we have in this case that $a \wedge a=0$, so this is a necessary condition to be decomposable which turns out to be sufficient according to the following Theorem.

Theorem 4.1. Let $a \in \bigwedge^{2} V, a \neq 0$. Then $a$ is decomposable if and only if $a \wedge a=0$.

The proof of this Theorem can be found in the Appendix.

4.1.1. Example. The bivector $\alpha=v_{0} \wedge v_{1}+v_{0} \wedge v_{3}-v_{1} \wedge v_{3}+v_{2} \wedge v_{1}+v_{2} \wedge v_{3}$ satisfies $\alpha \wedge \alpha=0$, and the reader can readily verify that $\alpha=\left(v_{0}-v_{1}+v_{2}\right) \wedge\left(v_{1}+v_{3}\right)$.

Let us write the condition $a \wedge a=0$ in the case $V=\mathbf{C}^{4}$. Given

$$
a=\sum_{0 \leq i<j \leq 4} a_{i j} v_{i} \wedge v_{j} \in \bigwedge^{2} V
$$

we have that

$$
a \wedge a=2\left(a_{01} a_{23}-a_{02} a_{13}+a_{03} a_{12}\right) v_{0} \wedge v_{1} \wedge v_{2} \wedge v_{3} .
$$

Therefore the necessary and sufficient condition for a two-vector to be decomposable is that $a_{01} a_{23}-a_{02} a_{13}+a_{03} a_{12}=0$, which is a non-degenerated quadratic expression in the six variables $a_{i j}, 0 \leq i<j \leq 4$. So, passing to projective spaces, we obtain the following result:

Theorem 4.2. The set of lines of $\mathbf{P}^{3}, \mathbb{G}(1,3)$, can be identified with a nondegenerated quadric $\Omega \subset P\left(\bigwedge^{2} V\right)$. This quadric is called the Klein quadric and has equation $a \wedge a=0$.

We have an analogous representation of $\mathbb{G}(1,3)$ as the quadric given by those $\alpha \in \bigwedge^{2} V^{*}$ such that $\alpha \wedge \alpha=0$. The six numbers $a_{i j}, 0 \leq i<j \leq 4$ are known as the Plücker coordinates of the line represented by the corresponding bivector. 
4.2. Matrix expression of the Klein quadric. Let us list the three algebraic equivalent objects from which the Klein quadric can be defined. We recall that a quadric can be equivalently seen as a quadric form, a symmetric bilinear form, and a symmetric mapping between a vector space and its dual (see 8.2 in the Appendix). As usual, we take a volume form $\mathfrak{V}=v_{0} \wedge v_{1} \wedge v_{2} \wedge v_{3}$ so we can identify $\wedge^{4} V \equiv \mathbf{C}$.

(1) The quadratic form is given by $\Omega(a)=a \wedge a$ for any bivector $a \in \wedge^{2} V$.

(2) The bilinear form is given by $\Omega(a, b)=a \wedge b$. This form of the Klein quadric is particularly interesting, since given decomposable bivectors $a, b \in \bigwedge^{2} V$, $a \wedge b=0$ if and only the lines they represent intersect. To see this it is enough to note that if $\alpha \wedge \beta=A(a \wedge b)$, we have that

$$
\begin{aligned}
a \wedge b=0 & \Longleftrightarrow \quad \text { (by definition of } A \text {, see formula }(3)) \\
(A a)(b)=0 & \Longleftrightarrow \text { (by formula in subsection 2.6) } \\
C_{[2]}(b \otimes A a)=0 & \Longleftrightarrow \text { The two lines intersect (due to Theorem 3.1. } \\
& \text { See also example 3.3.2). }
\end{aligned}
$$

(3) To define the polar form $\Omega: \bigwedge^{2} V \rightarrow \bigwedge^{2} V^{*}$ it is enough to know how $\Omega(a)$ acts on $\bigwedge^{2} V=\left(\bigwedge^{2} V^{*}\right)^{*}$. The rule is that $\Omega(a)(b)=\Omega(a, b)=a \wedge b$, but this is just the definition of the operator $A$ defined in section (3.2), so $\Omega$ coincides with the operator $A: \bigwedge^{2} V \rightarrow \bigwedge^{2} V^{*}$.

If we take the basis

$$
B \wedge^{2} V=\left\{v_{0} \wedge v_{1}, v_{0} \wedge v_{2}, v_{0} \wedge v_{3}, v_{1} \wedge v_{2}, v_{3} \wedge v_{1}, v_{2} \wedge v_{3}\right\}
$$

of $\bigwedge^{2} V$ and its dual basis

$$
B_{\wedge^{2} V^{*}}=2\left\{v_{0}^{*} \wedge v_{1}^{*}, v_{0}^{*} \wedge v_{2}^{*}, v_{0}^{*} \wedge v_{3}^{*}, v_{1}^{*} \wedge v_{2}^{*}, v_{3}^{*} \wedge v_{1}^{*}, v_{2}^{*} \wedge v_{3}^{*}\right\}
$$

we see, according to Example 3.2.1 that the matrix of the Klein quadric is

$$
M_{A}=\frac{1}{2}\left(\begin{array}{cccccc}
0 & 0 & 0 & 0 & 0 & 1 \\
0 & 0 & 0 & 0 & 1 & 0 \\
0 & 0 & 0 & 1 & 0 & 0 \\
0 & 0 & 1 & 0 & 0 & 0 \\
0 & 1 & 0 & 0 & 0 & 0 \\
1 & 0 & 0 & 0 & 0 & 0
\end{array}\right) .
$$

From now on we will refer only to this basis when we talk about the matrix of the Klein quadric.

Interpreting a column 6-vector $r$ as the coordinates of a bivector $a_{r}$ with respect to the basis $B \wedge^{2} V$, we can compute the coordinates of the bicovector $A\left(a_{r}\right)$ with respect to the basis $B \bigwedge^{2} V^{*}$ as the column vector $M_{A} r$. To check that $a_{r}$ is decomposable, one must verify that $r^{T} M_{A} r=0$, and to test if the line given by $r$ intersects $r^{\prime}$, one must check that $r^{\prime T} M_{A} r=0$. Similar computations can be carried out with covectors.

4.3. Interpretation of the tangent hyperplanes to the Klein quadric. The decomposable bivectors on the tangent hyperplane at a point of the Klein quadric have the following interesting interpretation:

Theorem 4.3. Given a decomposable bivector $a \in \bigwedge^{2} V$ representing a line $r$, the set of lines intersecting $r$ is given by the intersection of the tangent hyperplane to the Klein quadric at $[a] \in P\left(\bigwedge^{2} V\right)$ with the Klein quadric itself.

Proof. Let $a=u \wedge v$ represent a point of the Klein quadric. From the definition of this quadric and subsection 8.2, we have that the points of the tangent hyperplane at $a$ are those bivectors $a^{\prime}$ such that $a \wedge a^{\prime}=0$. The bivector $a^{\prime}$ represents a 
point of the Klein quadric if and only if it is decomposable, i.e., $a^{\prime}=u^{\prime} \wedge v^{\prime}$. But $a \wedge a^{\prime}=u \wedge v \wedge u^{\prime} \wedge v^{\prime}=0$ if and only if the vectors $u, v, u^{\prime}$ and $v^{\prime}$ are linearly dependent, and this happens if and only if the two lines represented by $a$ and $a^{\prime}$ intersect.

4.3.1. Example. Let us consider the line $r$ determined by the vectors $u=v_{0}+2 v_{1}+$ $v_{3}$ and $v=v_{1}+3 v_{2}$. The tangent hyperplane to the Klein quadric at $u \wedge v$ is given by those bivectors $a^{\prime}$ such that $u \wedge v \wedge a^{\prime}=0$ (see Appendix 8.2). If

$$
a^{\prime}=x_{01} v_{0} \wedge v_{1}+x_{02} v_{0} \wedge v_{2}+x_{03} v_{0} \wedge v_{3}+x_{12} v_{1} \wedge v_{2}+x_{13} v_{1} \wedge v_{3}+x_{23} v_{2} \wedge v_{3}
$$

then $u \wedge v \wedge a^{\prime}=0$ is equivalent to

$$
x_{23}-3 x_{13}+6 x_{03}+x_{02}-3 x_{01}=0 .
$$

Therefore the bivectors representing lines that intersect $r$ are those verifying the equation above together with $a^{\prime} \wedge a^{\prime}=0$, i.e.,

$$
x_{01} x_{23}-x_{02} x_{13}+x_{03} x_{12}=0 .
$$

4.4. Linear subspaces of the Klein quadric. There are only four kinds of linear subspaces of the Klein quadric: points, lines and $\alpha$ and $\beta$-planes. A point of the Klein quadric corresponds, as we have seen, with a line of $\mathbf{P}^{3}$. The lines of $\mathbf{P}^{3}$ passing through a fixed point represented by some $a \in V$, which is called vertex, are represented by the bivectors of the form $a \wedge b$, and these bivectors represent a plane of $\mathbf{P}^{5}$ contained in the Klein quadric, which is called an $\alpha$-plane. Besides, the lines contained in a given plane of $\mathbf{P}^{3}$ are linear combinations of three bivectors $a \wedge b$, $b \wedge c$ and $c \wedge a$, which represents three coplanar lines of $\mathbf{P}^{3}$. These linear combinations give a plane of the Klein quadric which is called a $\beta$-plane. Finally, the lines of the Klein quadric represent pencils of lines of $\mathbf{P}^{3}$, i.e., sets of lines passing through a point and contained simultaneously in a plane. They are therefore intersections of an $\alpha$-plane with a $\beta$-plane when this intersection is non-empty, i.e., when the vertex of the $\alpha$-plane lies on the base plane of the $\beta$-plane. These exhaust all the linear subspaces of the Klein quadric (see [8, Chapter 22]).

\section{The Absolute Line QuadriC}

Let us denote by $Q$ the dual absolute quadric (DAQ) [20] [9], which is given by the planes of $\mathbf{P}^{3}$ which are tangent to the absolute conic, including the plane at infinity itself. Since $Q \subset \mathbf{P}^{3^{*}}$, we can see it as a mapping $Q: V^{*} \rightarrow V$ (see 8.2) which, geometrically, is interpreted as the mapping sending to each plane of $\mathbf{P}^{3}$, different of the plane at infinity, a vector orthogonal to it. The quadric $Q$ happens to be of rank three and its kernel as a mapping is the plane at infinity itself. It is well known that the Euclidean structure of the space can be recovered from, and in fact is equivalent to, the knowledge of $Q$. We will call a basis of $V,\left\{v_{0}, v_{1}, v_{2}, v_{3}\right\}$ Euclidean when it is adapted to $Q$ in the sense that $Q\left(v_{i}^{*}\right)=v_{i}, i=0,1,2$ and $Q\left(v_{3}^{*}\right)=0$. Note that the plane at infinity is represented by the covector $v_{3}^{*}$ or by the three-vector $v_{0} \wedge v_{1} \wedge v_{2}$.

The DAQ extends to a linear mapping $\tilde{Q}: \bigwedge^{2} V^{*} \rightarrow \bigwedge^{2} V$ defined by

$$
\tilde{Q}(\alpha \wedge \beta)=(Q \alpha) \wedge(Q \beta) .
$$

This is a well-defined linear mapping as an immediate consequence of the universal property of exterior algebra (see 8.3 in the Appendix). The geometrical interpretation of this mapping is the following: If $\alpha \wedge \beta$ is the bicovector representing a line $r$ of $\mathbf{P}^{3}$, the bivector $(Q \alpha) \wedge(Q \beta)$ represents the line at infinity including all the directions orthogonal to $r$ (since both $Q \alpha$ and $Q \beta$ are orthogonal directions 


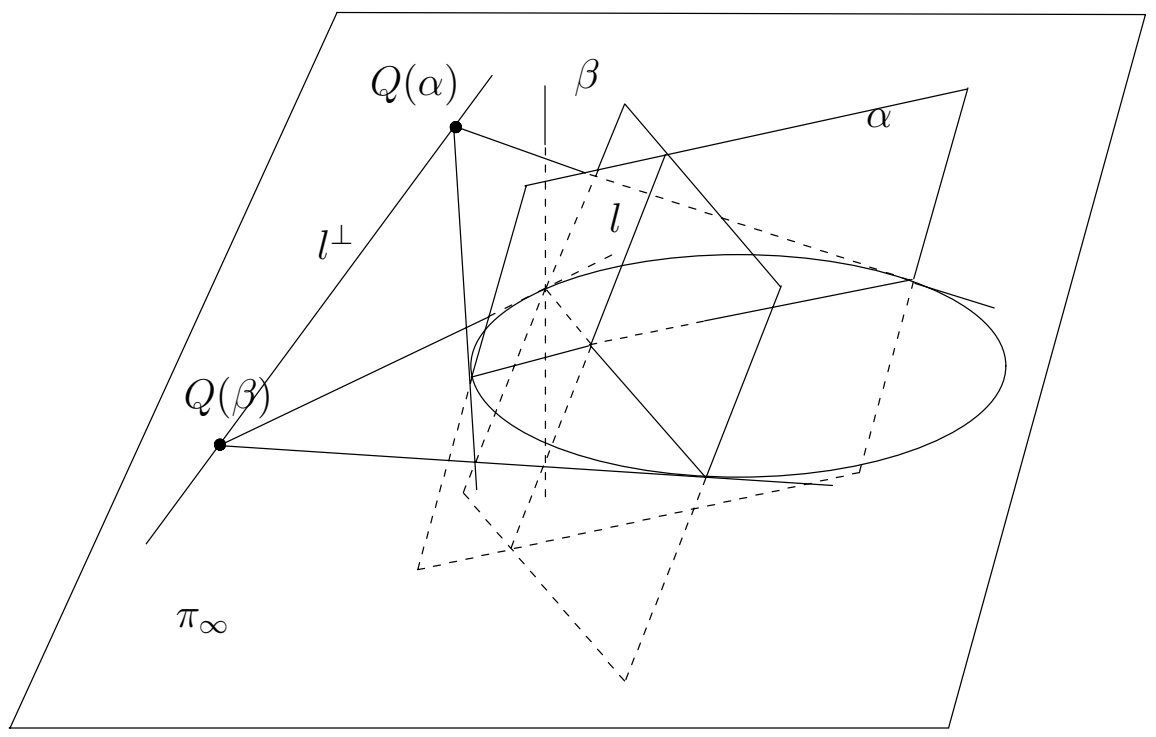

Figure 1. Obtaining the line of orthogonal directions to a line in $\mathbf{P}^{3}$.

to $r$ ). The mapping $\tilde{Q}$ will be called the absolute line quadric (ALQ). Note that definition (7) can be abbreviated as

$$
\tilde{Q}=Q \wedge Q
$$

so the ALQ is the wedge product of the DAQ with itself.

There are three properties of $\tilde{Q}$ that we want to remark:

(1) $\tilde{Q}$ is a rank-three quadric. To see this, let us take any Euclidean basis of $V,\left\{v_{i}\right\}$. The quadric $\tilde{Q}$ maps $v_{0}^{*} \wedge v_{1}^{*} \mapsto v_{0} \wedge v_{1}, v_{0}^{*} \wedge v_{2}^{*} \mapsto v_{0} \wedge v_{2}$, $v_{1}^{*} \wedge v_{2}^{*} \mapsto v_{1} \wedge v_{2}$ and any other basic bicovector is mapped to the null vector.

(2) Let us consider the polarity $\Omega: \bigwedge^{2} V \rightarrow \bigwedge^{2} V^{*}$ associated to the Klein quadric, which is an isomorphism since $\Omega$ is a full-rank quadric. The composite mapping $\tilde{Q} \Omega \tilde{Q}$ must vanish. To see this, note that $\Omega \tilde{Q}(\alpha \wedge \beta)$ is a bicovector representing a line in the plane at infinity, so it belongs to the kernel of $\tilde{Q}$.

(3) The quadratic form associated to $\tilde{Q}$ is given by the mapping $\tilde{Q}: \bigwedge^{2} V^{*} \rightarrow \mathbf{C}$, defined by

$$
\tilde{Q}(\alpha \wedge \beta):=(\alpha \wedge \beta)(Q(\alpha) \wedge Q(\beta)) .
$$

The zeroes of this quadric are those lines intersecting their own line of orthogonal directions and, therefore, they are those that intersect the absolute conic. To see this, remember that a decomposable bivector produces a zero when it acts on a decomposable bicovector if and only if the two lines they represent intersect (see subsection 4 ).

(4) Since $\tilde{Q}: \bigwedge^{2} V^{*} \rightarrow \bigwedge^{2} V$ is a rank-three mapping and its image is contained in the plane at infinity, it must coincide with it. Therefore this image is a $\beta$-plane.

5.1. Characterization of the quadrics $\tilde{Q}$. The following theorem characterizes the possible ALQs, in the sense that it describes the quadrics of $P\left(\bigwedge^{2} V^{*}\right)$ that are induced by rank-three quadrics of $V^{*}$. They happen to be exactly those that satisfy property (4) above. 
Theorem 5.1. A symmetric linear mapping $\tilde{Q}: \bigwedge^{2} V^{*} \rightarrow \bigwedge^{2} V$ is induced by a rank-three symmetric linear mapping $Q: V^{*} \rightarrow V$, in the sense that $\tilde{Q}(\alpha \wedge \beta)=$ $Q(\alpha) \wedge Q(\beta)$ for any $\alpha, \beta \in \bigwedge^{2} V^{*}$, if and only if $P\left(\tilde{Q}\left(\bigwedge^{2} V^{*}\right)\right)$ is a $\beta$-plane. In this case, $Q$ is uniquely given up to a sign.

From a practical point of view, the following equivalent characterization can be more useful.

Theorem 5.2. A symmetric linear mapping $\tilde{Q}: \bigwedge^{2} V^{*} \rightarrow \bigwedge^{2} V$ stems from a rank-three symmetric linear mapping $Q: V^{*} \rightarrow V$ if and only if the following three conditions hold true:

(1) The composite mapping

$$
\bigwedge^{2} V^{*} \stackrel{\tilde{Q}}{\longrightarrow} \bigwedge^{2} V \stackrel{\Omega}{\longrightarrow} \bigwedge^{2} V^{*} \stackrel{\tilde{Q}}{\longrightarrow} \bigwedge^{2} V
$$

vanishes.

(2) The rank of $\tilde{Q}$ is three.

(3) The image of $\tilde{Q}$ is not an $\alpha$-plane (conditions (1) and (2) above imply that $P\left(\tilde{Q}\left(\bigwedge^{2} V^{*}\right)\right)$ is either an $\alpha$-plane or a $\beta$-plane $)$.

Proofs of these theorems can be found in the Appendix.

Remark 5.1. From a practical point of view it is important to observe that condition (1) in Theorem 5.2 implies that the rank of $\tilde{Q}$ is at most three. To see this, just check that $\tilde{Q} \Omega \tilde{Q}=0$ implies

$$
\begin{aligned}
\operatorname{im} \Omega \tilde{Q} \subset \operatorname{ker} \tilde{Q} & \Rightarrow \\
\operatorname{dim} \operatorname{im} \Omega \tilde{Q} \leq \operatorname{dim} \operatorname{ker} \tilde{Q} & \Rightarrow(\text { Since } \Omega \text { is an isomorphism }) \\
\operatorname{rank} \tilde{Q} \leq 6-\operatorname{rank} \tilde{Q} & \Rightarrow \\
\operatorname{rank} \tilde{Q} \leq 3 . &
\end{aligned}
$$

5.2. Linear computation of the ALQ. In some applications it is possible to know a number of lines $\left\{r_{i}\right\}_{i=1}^{N}$ belonging to the ALQ and enough to determine it (see Section 6). One might hope to recover the ALQ obtaining the coefficients of its matrix by solving the linear system

$$
\tilde{Q}\left(r_{i}, r_{i}\right)=0, i=1, \ldots, N
$$

However, since also $\Omega\left(r_{i}, r_{i}\right)=0$ (Theorem 4.1), the result will include all the quadrics of the form $\alpha \tilde{Q}+\beta \Omega^{-1}$. This set of quadrics has been studied in [21] under the name of calibration pencil. In [21] it is also shown that they constitute all the possible solutions of the system. To obtain $\tilde{Q}$ from this pencil linearly we can use the following fact, whose proof can be found in the Appendix.

Theorem 5.3. Let $V$ be a four dimensional vector space and let $F: V^{*} \rightarrow V$ be a linear symmetric mapping (i.e., a quadric). Let $\tilde{F}: \bigwedge^{2} V^{*} \rightarrow \bigwedge^{2} V$ be the associated linear symmetric mapping defined by $\tilde{F}(\alpha \wedge \beta)=F(\alpha) \wedge F(\beta)$. Let $\Omega: \wedge^{2} V \rightarrow \wedge^{2} V^{*}$ be the Klein quadric. Then, the trace of the composition $\Omega \tilde{F}: \bigwedge^{2} V^{*} \rightarrow \bigwedge^{2} V^{*}$ is zero. 
If $F$ is given by a matrix $\left(f_{i j}\right)$, the matrix of $2 \tilde{F}$ with respect to the bases given by (5) and (6) will be

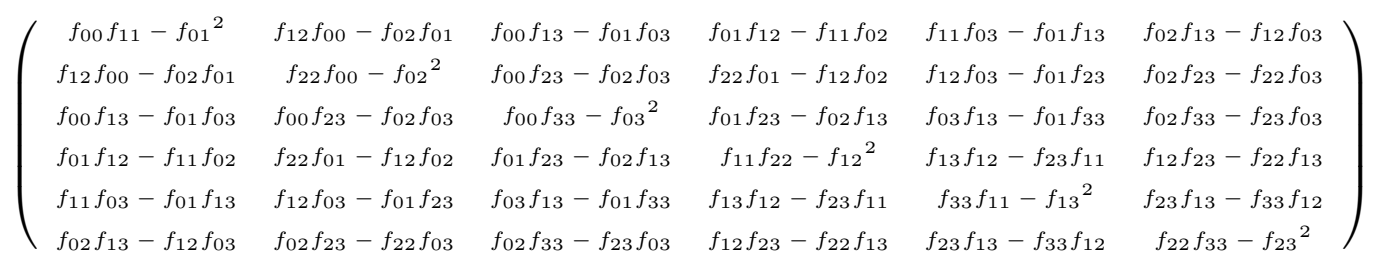

and it is straightforward to check that trace $\Omega \tilde{F}$, which is just the sum of the elements of the main antidiagonal of the matrix above, is zero.

We can therefore recover $\tilde{Q}$ from any linear combination $\alpha \tilde{Q}+\beta \Omega^{-1}$ noting that $\beta$ can be recovered as follows:

$$
\operatorname{trace}\left(\Omega\left(\alpha \tilde{Q}+\beta \Omega^{-1}\right)\right)=\operatorname{trace}(\alpha \Omega \tilde{Q}+\beta \operatorname{Id})=4 \beta \text {. }
$$

5.3. Recovering the DAQ from the ALQ. Algorithms to compute the ALQ from a projective calibration will be presented below. Here we will see how the DAQ can be recovered from the ALQ.

Suppose that $\tilde{Q}: \bigwedge^{2} V^{*} \rightarrow \bigwedge^{2} V$ is an ALQ, i.e., according to Theorem 5.1, it is a rank-three symmetric mapping such that its image is a $\beta$-plane, and let $\pi \in V^{*}$ be a representative of this plane. One can recover the value of $Q$ on a covector $\alpha \in V^{*}$ not a multiple of $\pi$ as follows: Take two auxiliary covectors $\beta$ and $\gamma$ also not multiple of $\pi$ and such that $\alpha, \beta$ and $\gamma$ are linearly independent. The bicovectors $\alpha \wedge \beta$ and $\alpha \wedge \gamma$ are representatives of two different lines of the plane represented by $\alpha$, and the images $\tilde{Q}(\alpha \wedge \beta)=Q(\alpha) \wedge Q(\beta), \tilde{Q}(\alpha \wedge \gamma)=Q(\alpha) \wedge Q(\gamma) \in \wedge^{2} V$ intersect at a point which must be a representative of $Q(\alpha)$. This intersection can be found, according to Theorem 3.1, computing the contraction $C_{[1]}\left(\tilde{Q}(\alpha \wedge \beta) \otimes A(\tilde{Q}(\alpha \wedge \gamma)) \in V \otimes V^{*}\right.$, which must be a multiple of $Q(\alpha) \otimes \pi$. The Lemma 5.4, proved in the Appendix, specifies this multiplicity factor.

Lemma 5.4. Let us consider a basis $\left\{v_{0}, \ldots, v_{3}\right\}$ of $V$, and let $u_{0}, u_{1}$ and $u_{2}$ be three linearly independent vectors of $V$. We have

$$
C_{[1]}\left(\left(u_{0} \wedge u_{1}\right) \otimes A\left(u_{0} \wedge u_{2}\right)\right)=\frac{1}{4} u_{0} \otimes \alpha
$$

where $\alpha$ is the covector

$$
\alpha=\sum_{i=0}^{3}(-1)^{i+1} M_{i} v_{i}^{*},
$$

and $M_{i}$ is the determinant of the $3 \times 3$ matrix obtained by suppressing the $i$-th row of the matrix $\left(u_{0} u_{1} u_{2}\right)$ given by the coordinates of the vectors $u_{i}$ in the given basis.

5.3.1. Example. Let us consider the vectors $u_{0}=2 v_{0}-v_{1}+v_{2}+3 v_{3}, u_{1}=3 v_{1}+3 v_{3}$ and $u_{2}=-v_{0}+v_{1}+4 v_{2}+3 v_{3}$. If we are given the bivectors

$$
\begin{aligned}
& u_{0} \wedge u_{1}=6 v_{0} \wedge v_{1}+2 v_{0} \wedge v_{3}-3 v_{1} \wedge v_{2}-10 v_{1} \wedge v_{3}+v_{2} \wedge v_{3} \\
& u_{0} \wedge u_{2}=v_{0} \wedge v_{1}+9 v_{0} \wedge v_{2}+9 v_{0} \wedge v_{3}-5 v_{1} \wedge v_{2}-6 v_{1} \wedge v_{3}-9 v_{2} \wedge v_{3} .
\end{aligned}
$$

let us see how we can recover multiples of $u_{0}$ and $\alpha$. We have that

$$
A\left(u_{0} \wedge u_{2}\right)=-9 v_{0} \wedge v_{1}+6 v_{0} \wedge v_{2}-5 v_{0} \wedge v_{3}+9 v_{1} \wedge v_{2}-9 v_{1} \wedge v_{3}+v_{2} \wedge v_{3}
$$

and

$$
C_{[1]}\left(\left(u_{0} \wedge u_{1}\right) \otimes A\left(u_{0} \wedge u_{2}\right)\right)=\frac{1}{4} \sum_{i, j=0}^{4} a_{i j} v_{i} \otimes v_{j}^{*}
$$


where the matrix $\left(a_{i j}\right)$ are entries of the matrix

$$
\Lambda=\left(\begin{array}{cccc}
-64 & -18 & -52 & 54 \\
32 & 9 & 26 & -27 \\
-32 & -9 & -26 & 27 \\
-96 & -27 & -78 & 81
\end{array}\right) .
$$

Knowing that $C_{[1]}\left(\left(u_{0} \wedge u_{1}\right) \otimes A\left(u_{0} \wedge u_{2}\right)\right)=\frac{1}{4} u_{0} \otimes \alpha$, we have $a_{i j}=\left(u_{0}\right)_{i} \alpha_{j}$, i.e.

$$
\Lambda=\left(\begin{array}{l}
\left(u_{0}\right)_{0} \\
\left(u_{0}\right)_{1} \\
\left(u_{0}\right)_{2} \\
\left(u_{0}\right)_{3}
\end{array}\right)\left(\begin{array}{llll}
\alpha_{0} & \alpha_{1} & \alpha_{2} & \alpha_{3}
\end{array}\right) .
$$

Therefore we can take any (non-zero) row of $\Lambda$ as a multiple of $\alpha$ and any column as a multiple of $u_{0}$. Adjusting constants, we can even recover $u_{0}^{\prime}=\lambda u_{0}$ and $\alpha^{\prime}=\lambda^{-1} \alpha$. In our case we can take, for example, $\alpha^{\prime}=-64 v_{0}^{*}-18 v_{1}^{*}-52 v_{2}^{*}+54 v_{3}^{*}$ and $u_{0}^{\prime}=(1 / 2) u_{0}$. The covector $\alpha$ provided by the lemma is given by $\alpha^{\prime} / 2$.

Lemma 5.4 provides a technique for the recovery of the rank-three $Q: V^{*} \rightarrow V$ (up to a sign) from its corresponding rank-three symmetric $\tilde{Q}: \bigwedge^{2} V^{*} \rightarrow \bigwedge^{2} V$. The problem is equivalent to that of finding the vectors $w_{i}=Q\left(v_{i}^{*}\right)$ from the data $\tilde{Q}\left(v_{j}^{*} \wedge v_{k}^{*}\right)=Q\left(v_{j}^{*}\right) \wedge Q\left(v_{k}^{*}\right)=w_{j} \wedge w_{k}$. If we select three indexes, say 0,1 and 2 , we can compute

$$
\begin{aligned}
& C_{[1]}\left(\left(w_{0} \wedge w_{1}\right) \otimes A\left(w_{0} \wedge w_{2}\right)\right)=\frac{1}{4} w_{0} \otimes \alpha_{0} \\
& C_{[1]}\left(\left(w_{1} \wedge w_{2}\right) \otimes A\left(w_{1} \wedge w_{0}\right)\right)=\frac{1}{4} w_{1} \otimes \alpha_{1} \\
& C_{[1]}\left(\left(w_{2} \wedge w_{0}\right) \otimes A\left(w_{2} \wedge w_{1}\right)\right)=\frac{1}{4} w_{2} \otimes \alpha_{2}
\end{aligned}
$$

and then note that $\alpha_{0}=\alpha_{1}=\alpha_{2}$ since we are cycling the vectors $w_{0}, w_{1}$ and $w_{2}$ and thus the columns of the matrices $M_{i}$ of Lemma 5.4, so they remain invariant. If the vectors $w_{0}, w_{1}$ and $w_{2}$ are linearly dependent, then $\alpha$ will be zero. In this case a new set of indexes must be employed. Since $Q$ is rank-three, at least one of the four possible selection of indexes will correspond to three linearly independent vectors, so that we will eventually come up with a non-zero $\alpha$. Let us assume that this is the case for the indexes 0,1 and 2 .

We can now find the coordinates of each vector $w_{i}, i=0,1,2$, as follows: If $w_{i}=\sum_{j=0}^{3} w_{i j} v_{j}$, we have that $w_{i} \otimes \alpha=\sum_{j, k=0}^{3} w_{i j} \alpha_{k} v_{j} \otimes v_{k}^{*}$. Then, since there must be at least one $\alpha_{k} \neq 0$, we have recovered the three vectors $w_{i}, i=0,1,2$, up to a common non-zero scale factor $\alpha_{k}$, using the method of example 5.3.1. This provides three columns of the matrix of $\alpha_{k} Q$, and, since this matrix is symmetric, this only leaves one unknown coefficient of it, which can be obtained from the null-determinant condition. The feasibility of this last computation is ensured by Lemma 8.1 in the Appendix.

If one is interested in the obtainment of $Q$ with the exact scale factor, this can be done up to a sign from the relation $\widetilde{\alpha_{k} Q}=\alpha_{k}^{2} \tilde{Q}$, which follows from the very definition of $\tilde{Q}$.

5.3.2. Dealing with noise. Note that in the noisy case, the contractions in equations (9) do not lead to decomposable tensors, i.e., elements of the form $a \otimes \alpha \in$ $V \otimes V^{*}$. Even if we approximate individually each tensor by a decomposable one, there is no guarantee that we will obtain the same covector $\alpha$. However, one can proceed as follows. Express each of the three tensors given by the left hand sides 
of equations (9) as a $4 \times 4$ matrix $A_{i}, i=0,1,2$, and compute a singular value decomposition (SVD)

$$
\left(\begin{array}{l}
A_{0} \\
A_{1} \\
A_{2}
\end{array}\right)=U_{12 \times 4} D_{4 \times 4} V_{4 \times 4}^{T},
$$

where $D$ is a diagonal matrix $D=\operatorname{diag}\left(d_{1}, d_{2}, d_{3}, d_{4}\right)$ and $d_{1} \geq d_{2} \geq d_{3} \geq d_{4} \geq 0$. Approximating $D$ by $\operatorname{diag}\left(d_{1}, 0,0,0\right)$ we obtain a decomposition

$$
\left(\begin{array}{l}
A_{0} \\
A_{1} \\
A_{2}
\end{array}\right) \approx\left(\begin{array}{l}
w_{0} \\
w_{1} \\
w_{2}
\end{array}\right) \alpha^{T}
$$

where the first factor is a $12 \times 1$ matrix corresponding to the vectors $w_{i}$ given by the first column of the matrix $U$ and the second factor is a $1 \times 4$ matrix corresponding to the coordinates of the covector $\alpha$ and is given by the first row of matrix $d_{1} V^{T}$.

5.4. Direct recovery of Euclidean coordinates from $\tilde{Q}$. The following theorem provides another way to recover the Euclidean structure of space from the ALQ. Now we see how to obtain directly the matrix of a coordinate change between a Euclidean reference and the projective reference employed. An equivalent result, from a different perspective, appeared in [15]. The proof that is provided in the Appendix is, up to the authors' knowledge, the first that can be found in the literature.

Theorem 5.5. Let $M_{\tilde{Q}}$ denote the matrix of the $A L Q$ in terms of bases defined as in (5) and (6). If $R$ is a $3 \times 6$ matrix such that $M_{\tilde{Q}}=R^{T} R$, then the rows of $R$ are the coordinates with respect to the basis given in equation (5) of the bivectors $w_{0} \wedge w_{1}, w_{1} \wedge w_{2}, w_{2} \wedge w_{0}$ where the vectors $w_{i}$ are such that if $w_{3}$ is any other 4-vector linearly independent with them, then $\left(\begin{array}{llll}w_{0} & w_{1} & w_{2} & w_{3}\end{array}\right)$ is the matrix of a change of coordinates from a Euclidean coordinate system.

From the proof a geometrical interpretation of the matrices $R$ and $R^{T}$ follows. The matrix $R$ is the matrix of the mapping

$$
\bigwedge^{2} V^{*} \stackrel{\tilde{Q}}{\longrightarrow} \operatorname{im} \tilde{Q}
$$

with respect to the basis $B \wedge^{2} V^{*}=2\left\{v_{0}^{*} \wedge v_{1}^{*}, v_{0}^{*} \wedge v_{2}^{*}, v_{0}^{*} \wedge v_{3}^{*}, v_{1}^{*} \wedge v_{2}^{*}, v_{3}^{*} \wedge v_{1}^{*}, v_{2}^{*} \wedge v_{3}^{*}\right\}$ associated with any basis of $V$ and the basis $B=\left\{u_{0} \wedge u_{1}, u_{1} \wedge u_{2}, u_{2} \wedge u_{0}\right\}$ of $\operatorname{im} \tilde{Q}$ where $\left\{u_{0}, u_{1}, u_{2}, u_{3}\right\}$ is an Euclidean basis of $V$. Note that $\operatorname{im} \tilde{Q}$ is the vector space associated to the $\beta$-plane corresponding to the lines of the plane at infinity. $2 R^{T}$ is the matrix of the inclusion

$$
\operatorname{im} \tilde{Q} \stackrel{i}{\longrightarrow} \bigwedge^{2} V
$$

with respect to the above-mentioned bases of $\operatorname{im} \tilde{Q}$ and $\bigwedge^{2} V$.

We can obtain such a factorization from a singular value decomposition $M_{\tilde{Q}}=$ $U D U^{t}$ :

$$
\begin{aligned}
M_{\tilde{Q}}=U D U^{t} & =\left(\begin{array}{ll}
A & C \\
B & D
\end{array}\right)\left(\begin{array}{ll}
\Delta & 0 \\
0 & 0
\end{array}\right)\left(\begin{array}{ll}
A^{t} & B^{t} \\
C^{t} & D^{t}
\end{array}\right) \\
& =\left(\begin{array}{l}
A \sqrt{\Delta} \\
B \sqrt{\Delta}
\end{array}\right)\left(\sqrt{\Delta} A^{t} \quad \sqrt{\Delta} B^{t}\right)=R R^{t},
\end{aligned}
$$

where $\Delta$ is a diagonal $3 \times 3$ matrix and $R$ is a rank-three $6 \times 3$ matrix. To recover the vectors $w_{i}$ from the rows of $R$ one can employ the procedure explained in subsection 5.3. 


\section{Applications to CAMERA autocalibration}

6.1. Camera model and motivation of the autocalibration technique. The above developed theory has an immediate application to camera autocalibration. We assume that cameras are modeled [9] by the equation $\mathbf{q} \sim P \mathbf{Q}$, where $\mathbf{Q}=$ $(x, y, z, t)^{T}$ denotes the Euclidean homogeneous coordinates of a spatial point, $\mathbf{q}=$ $(u, v, w)^{T}$ denotes the homogeneous coordinates of an image point, and $P$ is the $3 \times 4$ matrix $P=K(R \mid-R \mathbf{t})$. The intrinsic parameter matrix $K$ is given by

$$
K=\left(\begin{array}{ccc}
\alpha_{u} & -\alpha_{u} \cot \theta & u_{0} \\
0 & \alpha_{v} / \sin \theta & v_{0} \\
0 & 0 & 1
\end{array}\right)
$$

where $u_{0}$ and $v_{0}$ are the affine coordinates of the principal point, $\alpha_{u}$ and $\alpha_{v}$ are the pixel scale factors and $\theta$ is the skew angle between the axes of the pixel coordinates. We denote by $\tau=\alpha_{u} / \alpha_{v}$ the pixel aspect ratio. The matrix $R$ is a rotation matrix which gives the camera orientation, and $\mathbf{t}$ are the coordinates of the camera center.

We recall here [9] that it is possible to obtain a projective calibration only from image point correspondences. This means that, given a set of projected points $\mathbf{q}_{i j}$ obtained with $N$ cameras, $N \geq 2$, we can obtain a set of matrices $\hat{P}_{i}$ and a set of point coordinates $\hat{\mathbf{Q}}_{j}$ such that $\mathbf{q}_{i j} \sim \hat{P}_{i} \hat{\mathbf{Q}}_{j}$, where $\hat{P}_{i}=P_{i} H^{-1}$ and $\hat{\mathbf{Q}}_{j}=H \mathbf{Q}_{j}$ for some non-singular $4 \times 4$ matrix $H$.

Euclidean calibration can be defined as the obtainment of a matrix $H$ changing the projective coordinates of a given projective calibration to some Euclidean coordinate system, i.e., one in which the absolute conic has equations $x^{2}+y^{2}+z^{2}=$ $t=0$.

If the camera aspect ratio $\tau$ and skew $\theta$ are known, the affine coordinate transformation in the image given by the matrix

$$
K_{c}=\left(\begin{array}{ccc}
1 & \tau \cos \theta & 0 \\
0 & \tau \sin \theta & 0 \\
0 & 0 & 1
\end{array}\right)
$$

transforms the internal parameters matrix into

$$
K^{\prime}=K_{c} K=\left(\begin{array}{ccc}
\alpha^{\prime} & 0 & u_{0}^{\prime} \\
0 & \alpha^{\prime} & v_{0}^{\prime} \\
0 & 0 & 1
\end{array}\right),
$$

where $\alpha^{\prime}=\alpha_{u}, u_{0}^{\prime}=u_{0}+\tau \cos \theta v_{0}$ and $v_{0}^{\prime}=\tau \sin \theta v_{0}$. The matrix $K^{\prime}$ corresponds to the internal parameters matrix of a camera with square pixels. Therefore, if the pixel shape is known, we can always apply this transformation, solve the autocalibration problem for the case of square pixel cameras and finally apply the inverse transformation $K_{c}^{-1}$ to the estimated $K^{\prime}$ matrices. Therefore, without loss of generality, we will assume that we are dealing with square pixel cameras.

We can now introduce the geometric motivation of our method. The basic observation on which it is based is that the retroprojected lines of image points $(1, \pm i, 0)^{T}$ intersect the absolute conic, and therefore belong to the ALQ. To check this, note that if $\mathbf{Q}=(x, y, z, 0)^{T}$ are the coordinates of the intersection of one of these two lines with the plane at infinity $t=0$, we have that

$$
\begin{aligned}
(1, \pm i, 0)^{T} & \sim P \mathbf{Q}=K R(x, y, z)^{T}, \text { so } \\
(x, y, z)^{T} & \sim R^{T} K^{-1}(1, \pm i, 0)^{T}, \text { and then } \\
x^{2}+y^{2}+z^{2} & =(x, y, z)(x, y, z)^{T}=0 .
\end{aligned}
$$


If the aspect ratio is unknown but the skew is zero, an analogous computation shows that the lines obtained by back-projecting points $(1,0,0)$ and $(0,1,0)$ of each camera are orthogonal.

In any of these situations the use of the ALQ will permit to upgrade the projective calibration to an Euclidean calibration with linear algorithms. But first we need the following result (whose Grassmann-Cayley algebra version can be found in $[6$, p. 183]) in order to compute backprojections of image points.

Theorem 6.1. Let $P$ be a projection matrix with rows $v_{i}^{*} \in V^{*}, i=0,1,2$. Then the back-projected line of a given image point $\left(x_{0}, x_{1}, x_{2}\right)$ is represented by the bicovector

$$
\alpha=x_{0} v_{1}^{*} \wedge v_{2}^{*}+x_{1} v_{2}^{*} \wedge v_{0}^{*}+x_{2} v_{0}^{*} \wedge v_{1}^{*} .
$$

Proof. Let $v_{3}^{*} \in V^{*}$ be such that $\left\{v_{i}^{*}\right\}_{i=0}^{3}$ is a basis of $V^{*}$, and let $\left\{v_{i}\right\}_{i=0}^{3}$ be the associated predual basis of $V$. We compute a bivector representing the same line by applying the $A^{-1}$ operator according to the table in example 3.2.1, obtaining

$$
A^{-1}(\alpha)=x_{0} v_{0} \wedge v_{3}+x_{1} v_{1} \wedge v_{3}+x_{2} v_{2} \wedge v_{3}=\left(x_{0} v_{0}+x_{1} v_{1}+x_{2} v_{2}\right) \wedge v_{3} .
$$

Thus this is the line defined by points $x_{0} v_{0}+x_{1} v_{1}+x_{2} v_{2}$ and $v_{3}$. The first one is a space point that projects onto image point $\left(x_{0}, x_{1}, x_{2}\right)$ (to check it, just apply the projection equation and the definition of dual basis). The second is the camera center, since, again by the definition of dual basis, it belongs to the three planes represented by the covectors $v_{i}^{*}, i=0,1,2$.

6.2. Linear algorithms. Now we propose a linear method for finding the Euclidean structure of space from a set of $N \geq 10$ cameras with known skew and aspect ratio and varying focal length and principal point, based on the preceding analysis. We assume that a projective calibration has already been computed.

(1) Use the knowledge of the skew angle and aspect ratio of each camera to change the retinal coordinates so that the intrinsic parameter matrices have the form (11).

(2) Back-project the points $(1, \pm i, 0)$ computing the bicovectors representing the corresponding lines $r_{k}, \bar{r}_{k}$, as indicated in Theorem 6.1.

(3) Obtain the quadric $\tilde{Q}$ by solving the linear homogeneous system

$$
\left\{\begin{array}{l}
\tilde{Q}\left(r_{k}, r_{k}\right)=0 \\
\tilde{Q}\left(\bar{r}_{k}, \bar{r}_{k}\right)=0, k=1, \ldots, N \\
\operatorname{trace}(\Omega \tilde{Q})=0
\end{array}\right.
$$

Note that $\tilde{Q}$, as a symmetric $6 \times 6$ matrix, depends on 21 parameters defined up to scale. Each camera provides two linear equations, so $N=10$ cameras, resulting in $2 N+1=21$ equations, is the minimum number required in order to have, generically, an (over)determined system.

(4) Obtain from $\tilde{Q}$ the Euclidean calibration either by computing the DAQ, as explained in subsection 5.3 or by a direct recovery of an Euclidean coordinate system, as indicated in subsection 5.4.

As previously mentioned, if the skew is zero but the aspect ratio is unknown, a similar algorithm is possible in which one considers the back-projections of the points $(1,0,0)$ and $(0,1,0)$. In this case, a minimum of 20 cameras is required (cf. $[15])$.

6.3. Non-linear algorithm. The performance of linear algorithms is limited by the fact that their search for the matrix of $\tilde{Q}$ is not directly performed on the actual algebraic variety of the possible solutions, but on a linear space that contains this variety, i.e., the space of symmetric matrices with zero antitrace. 
Now we consider a technique to obtain a result on the actual solution space. In practice this leads to a nonlinear optimization problem that will benefit from the knowledge of an approximate initial solution provided by the linear method. In this context the usefulness of the linear algorithm will essentially depend on its ability to provide an approximate solution close enough to the the true solution, so that the subsequent nonlinear optimization converges to the optimal local minimum.

The proposed non-linear algorithm addresses the minimization of the cost function

$$
C(\tilde{Q})=\sum_{i}\left|\tilde{Q}\left(r_{i}, r_{i}\right)\right|^{2}+\sum_{i}\left|\tilde{Q}\left(\bar{r}_{i}, \bar{r}_{i}\right)\right|^{2}
$$

where the $r_{i}, \bar{r}_{i}$, have been defined in step (2) of the previous linear algorithm. The minimization of this cost function is constrained by the relationships

$$
\tilde{Q} \Omega \tilde{Q}=0,\|\tilde{Q}\|^{2}=1
$$

where $\|\cdot\|$ denotes the Frobenius norm.

The implementation essentially consists in the application of sequential quadratic programming (SQP) [7] to the minimization of the quadratic cost function $C$ over the space of $6 \times 6$ symmetric matrices with zero antitrace.

The SQP algorithm operates as follows.

(1) Apply the linear algorithm given in 6.2 to obtain initial values of the ALQ coefficients. Obtain from them the DAQ using the technique in 5.3 and from it again the ALQ using (8). We term $\tilde{Q}^{(0)}$ the resulting ALQ.

(2) Apply the following basic iteration until convergence of the $\operatorname{costs} C\left(\tilde{Q}^{(k)}\right)$ is achieved.

(a) Substitute the quadratic constraints (12) by their first order approximations around the available approximate solution $\tilde{Q}^{(k)}$.

(b) Solve exactly the resulting constrained optimization problem (quadratic cost function with linear constraints), obtaining $\tilde{Q}^{(k+1)}$.

6.4. Experimental results. The previously described algorithms have been tested with synthetic data in a series of experiments involving the reconstruction of a set of 100 points from their projections in 10 to 40 images taken with uncalibrated cameras with varying parameters. The $3 \mathrm{D}$ points lie close to the origin of coordinates of an Euclidean reference and the cameras are located at random positions lying approximately over a sphere centered at the origin and roughly pointing towards it, so that the set of projected points is approximately centered in the virtual CCD. Skew angle $\theta$ and aspect ratio $\tau$ vary with uniform distributions with respective averages $\pi / 2$ and 1 and maximum deviations of $\pm 10 \%$ from the average. Normalized focal length for each camera $\alpha$ is selected at random with a uniform distribution centered at $\alpha=3780$ with a maximum deviation of $\pm 10 \%$ from this value. The principal point is obtained from a uniform distribution with support in the square $[2560 / 4,1920 / 4]$. With these parameters the projected point coordinates have values within the square of sides $[-1500,1500]$ and, in each image, the points are contained inside a square of side 1500 pixels. After computing the point projections, these are perturbed by the addition of zero-mean Gaussian noise with different variances.

The complete processing for each experiment consists of a projective calibration followed by the computation of the camera parameters by means of the proposed algorithms. Projective calibration is performed in four steps. Firstly, an homography with matrix $T=\operatorname{diag}(c, c, 1)$ (similarity transformation) is applied to all projected points so that a normalization of the coordinates is performed. The value $c$ is the one that makes equal to $\sqrt{2}$ the average distance from the transformed points to the origin in the first image. Secondly, the "Gold Standard" algorithm described 
in [9]) is applied to a pair of images to obtain the projective calibration of two cameras. Next, the previously computed 3D points are employed for the linear computation of the projection matrices for the remaining images so that an initial projective calibration is achieved in the projective reference previously obtained for two cameras (see [9, p. 167]). Finally, a global projective bundle adjustment is performed (see [9, p. 423]).

For each camera configuration, Gaussian noise is added with typical deviations $\sigma$ between 0 and 5 pixels. Then the focal length relative error and the RMS error in the principal point are measured.

Figure 2 shows the results for 12 cameras as a function of noise typical deviation. Figure 3 shows the dependency of the results on both noise typical deviation and number of cameras. Observe that the higher computational cost of the non-linear algorithm is rewarded by a significantly better performance. Also note that in both cases the sensitivity on the number of cameras is meaningful between 10 and 15 cameras and afterwards it shows a saturation effect. Average computation times for a Matlab implementation running on a Pentium $4 \mathrm{CPU}$ at $2.66 \mathrm{GHz}$ are: $0.03 \mathrm{~s}$ for the initial projective calibration, $0.40 \mathrm{~s}$ for the projective bundle adjustment, $0.04 \mathrm{~s}$ for the initialization of the SQP algorithm by the linear technique, and $0.16 \mathrm{~s}$ for the SQP-based optimization.
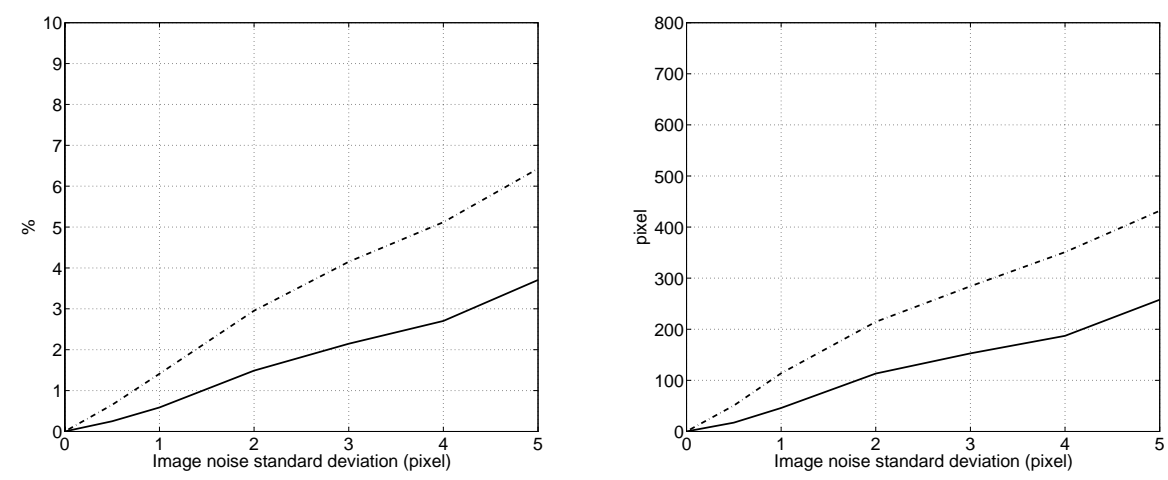

FiguRE 2. Error in focal length (left) and principal point (right) for 12 cameras as a function of the noise typical deviation (pixels). Continuous line: SQP algorithm; stippled line: linear algorithm.

\section{Conclusions And Future WORK}

We have shown how the Euclidean structure of space is encoded in the absolute line quadric (ALQ), given by the lines intersecting the absolute conic. The adequacy of exterior algebra to deal with linear subspaces of projective spaces has permitted (i) a clear and compact definition of the ALQ in terms of the dual absolute quadric (see (8)), (ii) a clean characterization of the ALQ (see theorem 5.1) and (iii)the design of techniques for the direct recovery of the information encoded by the ALQ (see theorem 5.5).

A linear and a non-linear algorithms for the autocalibration of cameras with known pixel shape and otherwise arbitrarily varying parameters have been tested with simulated data showing: (i) that the linear method is suitable to obtain an initial solution that can be later used as input for a non-linear algorithm and (ii) the good performance of the non-linear technique, with, for example, an average error in focal length of about $3 \%$ for noise standard deviation $\sigma=5$ and 15 cameras in 

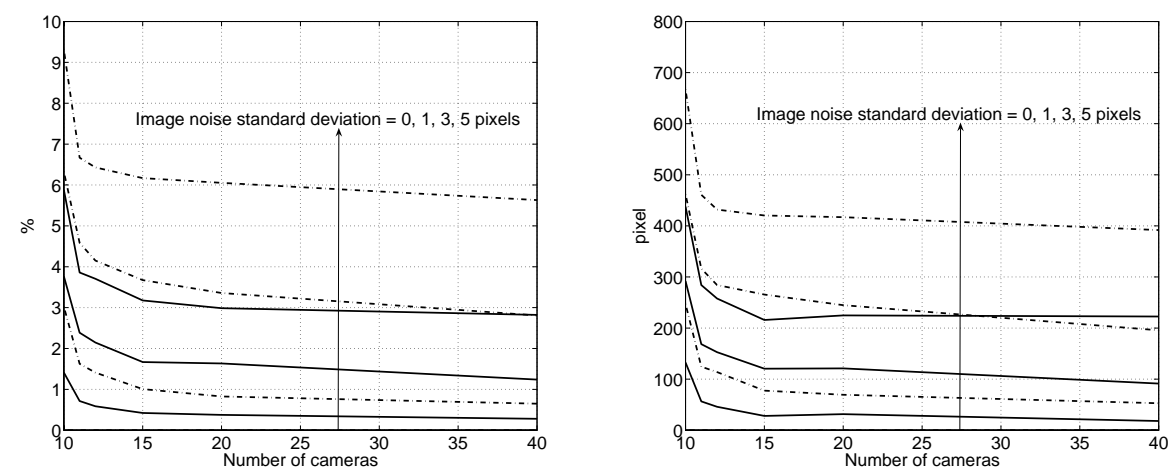

FiguRE 3. Error in focal length (left) and principal point (right) as a function of the number of cameras for noise typical deviations $\sigma=0,1,3$, and 5 pixels. Continuous line: SQP algorithm; stippled line: linear algorithm.

the experimental conditions explained in the text. The influence of the noise typical deviation and the number of cameras on the average errors have been empirically studied.

Among the remaining points for further study, we can mention the optimal approximation of an ALQ obtained by linear methods by a proper ALQ meeting all the constraints and the behavior of the described techniques in relation to degenerate camera configurations. Furthermore, the algorithmic possibilities of the ALQ are far from being completely exploited, including the performance of the algorithms with real images.

\section{APPENDIX}

8.1. Meet and join operators. The join operation $\nabla$ coincides the wedge product, i.e., $a \nabla b=a \wedge b$. The meet operator $\triangle$ can be introduced in several ways. The closest to our presentation is the following. Let $V$ be a real vector space in which a scalar product and an orientation are given. The Hodge star $*$ defined by $* v_{I}=(-1)^{\left|I, I^{\prime}\right|} v_{I^{\prime}}$ where $\left\{v_{i}\right\}$ is a positively oriented orthonormal basis and $I^{\prime}$ is the complement of $I$ in $\{1, \ldots, n\}$. It can be checked that this defines a linear mapping $*: \bigwedge^{p} V \rightarrow \bigwedge^{n-p+1} V$ independently of the chosen basis. Its relationship with the $A$ operator is the following. The scalar product induces a canonical isomorphism $\varphi: \bigwedge^{p} V^{*} \rightarrow \bigwedge^{p} V$ given by $\varphi\left(v_{I}^{*}\right)=v_{I}$. Then we have that $*=\varphi \circ A$, i.e., the following diagram is commutative:

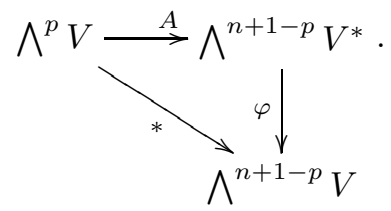

The meet operator can now be defined as a mapping which assigns to a pair $(a, b)$, $a \in \bigwedge^{p} V, b \in \bigwedge^{q} V$, the element

$$
a \triangle b=(-1)^{(p+q)(p+q-n-1)} *((* a) \wedge(* b)) \in \bigwedge^{p+q-n-1} V .
$$

It can be checked that this construction extends to the complexified vector space of $V$ (see [22, p. 156]). If $a$ and $b$ are representatives of subspaces $A$ and $B$, the meet operator gives a representative of $A \cap B$ if $A+B$ spans the whole space. So, 
for example, in $\mathbf{P}^{3}$ it fails when we try to check whether a given point lies or not on a line or to compute the intersection point of two coplanar lines.

8.2. Notation and generalities on quadrics. We will use three different ways to interpret algebraically a quadric $S$, defined up to a non-zero scalar factor.

(1) We can see it as a bilinear symmetric form $S: V \times V \rightarrow \mathbf{C}$.

(2) It can be seen as the quadratic form associated to the bilinear form given by $u \mapsto S(u, u)$. If we are given the quadratic form we can recover the bilinear form by means of the polarity identity, $S(u, v)=\frac{1}{2}(S(u+v, u+v)-$ $S(u, u)-S(v, v))$.

(3) A quadric can be given as a symmetric linear mapping $S: V \rightarrow V^{*}$, i.e., such that for any vector $u \in V$, the mapping $S(u): V \rightarrow \mathbf{C}$ satisfies $S(u)(v)=S(v)(u)$ for any vector $v \in V$. This third interpretation of a quadric is called the polar form. If we are given the bilinear symmetric form $S: V \times V \rightarrow \mathbf{C}$, we define the polar form $S: V \rightarrow V^{*}$ so that for $u \in V$ the element $S(u) \in V^{*}$ acts on vectors $v \in V$ by the rule $S(u)(v)=S(u, v)$. Conversely, given the bilinear symmetric form, we recover the polar form as $S(u)=S(u, \cdot)$. It is worth to note that the tangent hyperplane to the zero-set of the quadric at the point represented by $u_{0}$ is given by the linear form $S\left(u_{0}\right)$, i.e., it has equation $S\left(u_{0}, v\right)=0$.

Taking any basis $\left\{v_{i}\right\}$ of $V$, the matrix of $S$ is given by $S_{i j}=S\left(v_{i}, v_{j}\right)$. Its polar form, $S: V \rightarrow V^{*}$ is given by $S\left(v_{i}\right)=\sum_{j=0}^{n} S_{i j} v_{j}^{*}$.

8.3. Universal property of exterior algebra. The exterior product satisfies the following universal property: given any alternating multilinear mapping $f$ : $V \times \cdots \times V \rightarrow W$, there exists a unique linear mapping $\tilde{f}: \bigwedge^{k} V \rightarrow W$ such that $\tilde{f}\left(u_{1} \wedge \cdots \wedge u_{k}\right)=f\left(u_{1}, \ldots, u_{k}\right)($ see $[5$, p. 427]).

\subsection{Lemmas and proofs.}

Proof of equation (4). To check this, it is enough to verify that for any basis element $v_{J} \in \bigwedge^{n+1-p} V$ we have that $A v_{I}\left(v_{J}\right)=(-1)^{\left|I, I^{\prime}\right|} v_{I^{\prime}}^{*}\left(v_{J}\right)$. If $J$ is not a permutation of $I^{\prime}$ then both members are zero. In the other case, denoting by $(-1)^{\left|J \rightarrow I^{\prime}\right|}$ the signature of the permutation taking $J$ to $I^{\prime}$, we have that

$$
\left(A v_{I}\right)\left(v_{J}\right) \mathfrak{V}=v_{I} \wedge v_{J}=(-1)^{\left|J \rightarrow I^{\prime}\right|} v_{I} \wedge v_{I^{\prime}}=v_{I^{\prime}}^{*}\left(v_{J}\right)(-1)^{\left|I, I^{\prime}\right|} \mathfrak{V}
$$

since $v_{I^{\prime}}^{*}\left(v_{J}\right)=(-1)^{\left|J \rightarrow I^{\prime}\right|}$ and $v_{I} \wedge v_{I^{\prime}}=(-1)^{\left|I, I^{\prime}\right|} \mathfrak{V}$.

Proof of Theorem 4.1. Given a covector $\alpha \in V^{*}$ and a $p$-vector $a \in \bigwedge^{p} V$, we define the interior product $\alpha\lrcorner a=p C_{[1]}(\alpha \otimes a)$. It is easy to check that the interior product is a linear mapping carrying $p$-covectors into $(p-1)$-covectors and such that $\left.\alpha\lrcorner(a \wedge b)=(\alpha\lrcorner a) \wedge b+(-1)^{|b|} a \wedge(\alpha\lrcorner b\right)$. Let $\operatorname{dim} V=n+1$, and let us take any $\alpha \in V^{*}$. We have that

$$
\left.\left.0=\alpha\lrcorner(a \wedge a)=(\alpha\lrcorner a) \wedge a+(-1)^{|a|} a \wedge(\alpha\lrcorner a\right)=2(\alpha\lrcorner a\right) \wedge a,
$$

taking into account formula (2) and that $|a|=2$. If we find any $\alpha \in V^{*}$ such that $V \ni v=\alpha\lrcorner a \neq 0$, we are done since $v \wedge a=0$ implies that $a=v \wedge w$ for some $w \in V$. This last assertion is easily checked considering an adapted basis of $V$, $\left\{v_{i}\right\}$, such that $v_{0}=v$ and checking that the condition $v \wedge a=0$ implies that any coefficient $a_{i j}$ with $0<i<j$ in the decomposition $a=\sum_{i<j} a_{i j} v_{i} \wedge v_{j}$ must vanish, so we can factor out $v_{0}$.

Therefore it remains to prove that there exists some $\alpha \in V^{*}$ such that $\left.\alpha\right\lrcorner a \neq 0$. Let $\left.W^{*}=\left\{\alpha \in V^{*}: \alpha\right\lrcorner a=0\right\}$. Clearly $W^{*}$ is a vector subspace of $V^{*}$. We assert that $\operatorname{dim} W^{*}=p \leq(n+1)-2$. To see this, it is enough to consider again a cobasis 
adapted to $W^{*}$, say $\left\{v_{i}^{*}\right\}$, such that $W^{*}$ is represented by $v_{0}^{*} \wedge \cdots \wedge v_{p-1}^{*}$. Let $\left\{v_{i}\right\}$ be its dual basis. For any of the $v_{k}^{*} \in W^{*}$ we have that

$$
\left.v_{k}^{*}\right\lrcorner a=-\sum_{0 \leq i<k} a_{i k} v_{i}+\sum_{k<j \leq n} a_{k j} v_{j}=0
$$

so any coefficient $a_{i k}$ or $a_{k j}, 0 \leq k \leq p-1,0 \leq i, j \leq n$, must vanish, i.e., $a$ can be written using exclusively the last $n+1-p$ vectors $v_{p}, \ldots, v_{n}$. But since $0 \neq a \in \bigwedge^{2} V$, we need at least two vectors of the basis to build $a$, so $n+1-p \geq 2$, i.e., $p \leq n-1$. Therefore $W^{*}$ does not exhaust $V^{*}$ and there exists $\alpha \in V^{*}$ such that $\alpha\lrcorner a \neq 0$.

Proof of Theorem 5.1. First we check that the conditions are necessary. If $Q$ : $V^{*} \rightarrow V$ is a symmetric linear mapping of rank three, $P\left(Q\left(V^{*}\right)\right)$ is a plane of $\mathbf{P}^{3}$ and, consequently, the image of its associate mapping, $P\left(\tilde{Q}\left(\bigwedge^{2} V^{*}\right)\right)$, corresponds to the lines contained in this plane, i.e., it is a $\beta$-plane.

Let us check that the condition is also sufficient, so let $\tilde{Q}: \bigwedge^{2} V^{*} \rightarrow \bigwedge^{2} V$ be a symmetric mapping whose image is a $\beta$-plane (we call it $\tilde{Q}$ although we do not know yet whether it is associated to some $Q$ ). Let $v_{0}^{*}$ be a representative of the plane of $\mathbf{P}^{3}$ corresponding to this $\beta$-plane, and let $B^{*}=\left\{v_{0}^{*}, \ldots, v_{3}^{*}\right\}$ be a basis of $V^{*}$. Let $B=\left\{v_{0}, \ldots, v_{3}\right\}$ be its dual basis. Therefore $\tilde{Q}\left(\bigwedge^{2} V^{*}\right)$ is spanned by $\left\{v_{1} \wedge v_{2}, v_{2} \wedge v_{3}, v_{3} \wedge v_{1}\right\}$, and therefore, with respect to the bases $2\left\{v_{0}^{*} \wedge v_{1}^{*}, v_{0}^{*} \wedge\right.$ $\left.v_{2}^{*}, v_{0}^{*} \wedge v_{3}^{*}, v_{1}^{*} \wedge v_{2}^{*}, v_{2}^{*} \wedge v_{3}^{*}, v_{3}^{*} \wedge v_{1}^{*}\right\}$ and $\left\{v_{0} \wedge v_{1}, v_{0} \wedge v_{2}, v_{0} \wedge v_{3}, v_{1} \wedge v_{2}, v_{2} \wedge v_{3}, v_{3} \wedge v_{1}\right\}$, the matrix $M_{\tilde{Q}}$ of $\tilde{Q}$ has its three first rows equal to zero. Taking into account the symmetry of this matrix, we have

$$
M_{\tilde{Q}}=\left(\begin{array}{cc}
0_{3 \times 3} & 0_{3 \times 3} \\
0_{3 \times 3} & C
\end{array}\right)
$$

for some $3 \times 3$ nonsingular symmetric matrix $C$. Now let us consider any rank-three linear symmetric mapping $Q^{\prime}: V^{*} \rightarrow V$. If we impose that its image corresponds to $v_{0}^{*}$ and we take into account its symmetry, we see that its matrix with respect to the bases $B^{*}$ and $B$ will have the form

$$
N_{Q^{\prime}}=\left(\begin{array}{cc}
0_{1 \times 1} & 0_{1 \times 3} \\
0_{3 \times 1} & D
\end{array}\right)
$$

for some nonsingular symmetric $3 \times 3$ matrix $D$. A direct computation shows that $Q^{\prime}$ induces a symmetric linear mapping $V^{*} \wedge V^{*} \rightarrow V \wedge V$ with matrix

$$
N_{\tilde{Q}^{\prime}}=\left(\begin{array}{cc}
0_{3 \times 3} & 0_{3 \times 3} \\
0_{3 \times 3} & \operatorname{adj} D
\end{array}\right)
$$

Consequently, the mapping $Q$ given by matrix

$$
Q \equiv\left(\begin{array}{cc}
0_{1 \times 1} & 0_{1 \times 3} \\
0_{3 \times 1} & \sqrt{|C|} C^{-1}
\end{array}\right)
$$

(or its opposite as well) will induce the mapping $\tilde{Q}$.

Proof of Theorem 5.2. From Theorem 5.1 we know that the hypothesis of this Theorem is equivalent to the property that the image of $\tilde{Q}$ is a $\beta$-plane. Let us first consider the weaker condition that the image of $\tilde{Q}$ is a subset (necessary linear) of 
the Klein quadric. We have the following chain of equivalences:

$$
\begin{aligned}
& \tilde{Q}(\alpha) \text { belongs to the Klein quadric } \forall \alpha \in \bigwedge^{2} V^{*} \\
& \Longleftrightarrow \text { (definition of the Klein quadric) } \tilde{Q}(\alpha) \wedge \tilde{Q}(\alpha)=0 \forall \alpha \in \bigwedge^{2} V^{*} \\
&\Longleftrightarrow \text { (definition of } A) A(\tilde{Q}(\alpha))(\tilde{Q}(\alpha))=0 \forall \alpha \in \bigwedge^{2} V^{*} \\
&\Longleftrightarrow \text { (symmetry of } \tilde{Q})((\tilde{Q} A \tilde{Q}) \alpha)(\alpha)=0 \forall \alpha \in \bigwedge^{2} V^{*} \\
& \Longleftrightarrow \tilde{Q} A \tilde{Q}=0 .
\end{aligned}
$$

Therefore, to assure that the image of $\tilde{Q}$ is a plane of the Klein quadric, we must check that $\tilde{Q} A \tilde{Q}=0$ and that $\operatorname{rank} \tilde{Q}=3$. If this happens, its image must be either an $\alpha$-plane or a $\beta$-plane, so it is necessary to check also this, which is a discrete condition. This ends the proof of the Theorem.

Proof of Theorem 5.3. We recall that given any endomorphism of a vector space, $G: W \rightarrow W$, the trace of $G$ can be computed as $\operatorname{trace}(G)=\sum_{i} w_{i}^{*}\left(G\left(w_{i}\right)\right)$ for any basis $\left\{w_{i}\right\}$ of $W$. Let $\left\{v_{i}\right\}$ be a basis of $V$ adapted to $F$, in the sense that $F\left(v_{i}^{*}\right)=\lambda_{i} v_{i}$. Such a basis exists since $F$ is symmetric. Now we compute the trace of $\Omega \tilde{F}$ using the basis $\left\{2 v_{i}^{*} \wedge v_{j}^{*}, i<j\right\}$ of $\bigwedge^{2} V^{*}$, as

$$
\begin{aligned}
\sum_{i<j}\left(v_{i} \wedge v_{j}\right)\left(\Omega \tilde{F}\left(2 v_{i}^{*} \wedge v_{j}^{*}\right)\right)=0 & \Longleftrightarrow(\text { Definition of } \tilde{F}) \\
\sum_{i<j}\left(v_{i} \wedge v_{j}\right)\left(\Omega\left(F\left(v_{i}^{*}\right) \wedge F\left(v_{j}^{*}\right)\right)\right)=0 & \Longleftrightarrow(\text { Definition of } \Omega=A \text { in equation }(3)) \\
\sum_{i<j} \Omega\left(v_{i} \wedge v_{j}\right) \wedge \Omega\left(F\left(v_{i}^{*}\right) \wedge F\left(v_{j}^{*}\right)\right)=0 & \Longleftrightarrow(\text { The basis is adapted to } F) \\
\sum_{i<j} \Omega\left(v_{i} \wedge v_{j}\right) \wedge \Omega\left(\left(\lambda_{i} v_{i}\right) \wedge\left(\lambda_{j} v_{j}\right)\right)=0, &
\end{aligned}
$$

and the last equation holds since $\Omega$ transforms decomposable bivectors onto decomposable bivectors.

Proof of Lemma 5.4. Let $u_{3}$ be any vector such that the matrix $C=\left(\begin{array}{llll}u_{0} & u_{1} & u_{2} & u_{3}\end{array}\right)$ has determinant equal to one. Let $C^{*}=\left(\begin{array}{llll}u_{0}^{*} & u_{1}^{*} & u_{2}^{*} & u_{3}^{*}\end{array}\right)$ expressed in the basis $\left\{v_{i}^{*}\right\}$. Note that $C^{* t} C=I_{4}$, so $C^{*}=C^{-t}$. Since $\operatorname{det} C=1$, it follows that $u_{3}^{*}$ has coordinates $\left(-M_{0}, M_{1},-M_{2}, M_{3}\right)^{t}$. According to the table in 3.2.1, $A\left(u_{0} \wedge u_{2}\right)=$ $u_{3}^{*} \wedge u_{1}^{*}$, and

$$
C_{[1]}\left(\left(u_{0} \wedge u_{1}\right) \otimes A\left(u_{0} \wedge u_{2}\right)\right)=\frac{1}{4} u_{0} \otimes u_{3}^{*} .
$$

Therefore $\alpha=u_{3}^{*}=\left(-M_{0}, M_{1},-M_{2}, M_{3}\right)^{t}$.

Lemma 8.1. A symmetric $4 \times 4$ rank-three matrix is determined by any three linearly independent rows or columns.

Proof. Let $Q=\left(Q_{i j}\right)$ be such a matrix. Being $Q$ symmetric, it is clear that the knowledge of three rows or columns leaves only one unknown coefficient. Reordering coordinates, if necessary, we can assume that the first three columns are known, so the only unknown coefficient is $Q_{44}$. The condition $\operatorname{det} Q=0$ is a linear determined equation in this coefficient as long as $\operatorname{det} Q_{1 . .3,1 . .3} \neq 0$, so this is what should be proved.

Let us denote by $u_{i}$ the columns of $Q$. Being the first three columns linearly independent and $\operatorname{rank} Q=3$, there must exist a linear relation

$$
u_{4}=\lambda_{1} u_{1}+\lambda_{2} u_{2}+\lambda_{3} u_{3} \text {. }
$$


Let us see how the condition $\operatorname{det} Q_{1 . .3,1 . .3}=0$ leads us to a contradiction. Denoting by $\hat{u}_{i}$ the three-vector obtained removing the last coordinate of $u_{i}$, the condition $\operatorname{det} Q_{1 . .3,1 . .3}=0$ is equivalent to

$$
\operatorname{dim} \operatorname{span}\left\langle\hat{u}_{1}, \hat{u}_{2}, \hat{u}_{3}\right\rangle \leq 2 .
$$

This, together with equation (13) yields

$$
\operatorname{dim} \operatorname{span}\left\langle\hat{u}_{1}, \hat{u}_{2}, \hat{u}_{3}, \hat{u}_{4},\right\rangle \leq 2
$$

which contradicts, since $Q$ is symmetric, the linear independence of the first three columns.

Proof of Theorem 5.5. First let us prove that in any decomposition $M_{\tilde{Q}}=R^{T} R$, the matrix $R$ is of the form $R=N\left(\begin{array}{ll}P & Q\end{array}\right)$ where $\left(\begin{array}{ll}P & Q\end{array}\right)$ is some fixed rank-three matrix independent of $R$ and $N$ is an orthogonal matrix. Therefore, given two decompositions $M_{\tilde{Q}}=R^{T} R$ and $M_{\tilde{Q}}=\bar{R}^{T} \bar{R}$, there exists an orthogonal matrix $\bar{N}$ such that $\bar{R}=\bar{N} R$.

To see this, let us consider a $6 \times 6$ regular matrix $H$ that diagonalizes $M_{\tilde{Q}}$, i.e., such that

$$
H^{T} M_{\tilde{Q}} H=\left(\begin{array}{cc}
I_{3 \times 3} & 0_{3 \times 3} \\
0_{3 \times 3} & 0_{3 \times 3}
\end{array}\right) .
$$

Let $R_{0}:=\left(\begin{array}{ll}N & W\end{array}\right):=R H$. Let us check that $N$ is orthogonal and that $W=0$. We have

$$
H^{T} M_{\tilde{Q}} H=H^{T} R^{T} R H=R_{0}^{T} R_{0}=\left(\begin{array}{ll}
N^{T} N & N^{T} W \\
W^{T} N & W^{T} W
\end{array}\right)=\left(\begin{array}{ll}
I & 0 \\
0 & 0
\end{array}\right) .
$$

Therefore $N$ is indeed a $3 \times 3$ orthogonal matrix and $W=0$. So $R_{0}=\left(\begin{array}{ll}N & 0\end{array}\right)$, and $\operatorname{defining}\left(\begin{array}{ll}P & Q \\ S & T\end{array}\right):=H^{-1}$, we have that $R=R_{0} H^{-1}=N\left(\begin{array}{ll}P & Q\end{array}\right)$, as we wanted to see.

Now we are going to consider a particular geometrically meaningful decomposition $M_{\tilde{Q}}=R^{T} R$. So let us consider an ALQ, i.e., a symmetric mapping $\tilde{Q}: \bigwedge^{2} V^{*} \rightarrow \bigwedge^{2} V$ whose image is a $\beta$-plane, stemming from a DAQ $Q: V^{*} \rightarrow V$. We can see $\tilde{Q}$ as a composition

$$
\bigwedge^{2} V^{*} \stackrel{\tilde{Q}}{\longrightarrow} \operatorname{im} \tilde{Q} \stackrel{i}{\longrightarrow} \bigwedge^{2} V
$$

where $i$ is the inclusion mapping.

We consider an Euclidean basis of $V,\left\{u_{0}, u_{1}, u_{2}, u_{3}\right\}$. This basis produces an associated basis $B=\left\{u_{0} \wedge u_{1}, u_{1} \wedge u_{2}, u_{2} \wedge u_{0}\right\}$ of im $\tilde{Q}$.

The matrix of the mapping $\tilde{Q}: \bigwedge^{2} V^{*} \rightarrow \operatorname{im} \tilde{Q}$ with respect to the basis $B \wedge^{2} V^{*}=$ $2\left\{v_{0}^{*} \wedge v_{1}^{*}, v_{0}^{*} \wedge v_{2}^{*}, v_{0}^{*} \wedge v_{3}^{*}, v_{1}^{*} \wedge v_{2}^{*}, v_{3}^{*} \wedge v_{1}^{*}, v_{2}^{*} \wedge v_{3}^{*}\right\}$ associated with any basis of $V$ and the basis $B$ of $\operatorname{im} \tilde{Q}$ will be given by a $3 \times 6$ matrix

$$
R=\left(\begin{array}{ccc}
R_{01}^{01} & \cdots & R_{23}^{01} \\
R_{01}^{12} & \cdots & R_{23}^{12} \\
R_{01}^{20} & \cdots & R_{23}^{20}
\end{array}\right)
$$

of entries of the form $R_{i j}^{k l}=2\left(u_{k}^{*} \wedge u_{l}^{*}\right)\left(\tilde{Q}\left(2 v_{i}^{*} \wedge v_{j}^{*}\right)\right)$, by the very definition of dual basis.

Now let us consider the matrix of the inclusion mapping $\operatorname{im} \tilde{Q} \hookrightarrow \bigwedge^{2} V$ with respect to the bases $B$ and $B \wedge^{2} V=\left\{v_{0} \wedge v_{1}, v_{0} \wedge v_{2}, v_{0} \wedge v_{3}, v_{1} \wedge v_{2}, v_{3} \wedge v_{1}, v_{2} \wedge v_{3}\right\}$. 
The entries of this matrix are of the form $2\left(v_{i}^{*} \wedge v_{j}^{*}\right)\left(u_{k} \wedge u_{l}\right)$. We have

$$
\begin{aligned}
2\left(v_{i}^{*} \wedge v_{j}^{*}\right)\left(u_{k} \wedge u_{l}\right) & =(\text { since the basis is adapted to } Q) \\
2\left(v_{i}^{*} \wedge v_{j}^{*}\right)\left(Q\left(u_{k}^{*}\right) \wedge Q\left(u_{l}^{*}\right)\right) & =(\text { definition of } \tilde{Q}) \\
2\left(v_{i}^{*} \wedge v_{j}^{*}\right)\left(\tilde{Q}\left(u_{k}^{*} \wedge u_{l}^{*}\right)\right) & =2\left(u_{k}^{*} \wedge u_{l}^{*}\right)\left(\tilde{Q}\left(v_{i}^{*} \wedge v_{j}^{*}\right)\right) \quad(\text { since } \tilde{Q} \text { is symmetric) }
\end{aligned}
$$

so we conclude that the matrix of the inclusion is just $2 R^{T}$. Therefore the matrix $M_{\tilde{Q}}$ can be written as a product $M_{\tilde{Q}}=2 R^{T} R=(\sqrt{2} R)^{T}(\sqrt{2} R)$, as desired, and the rows of $R$ turn out to be the coordinates with respect to the basis $B \wedge^{2} V$ of the bivectors $u_{0} \wedge u_{1}, u_{1} \wedge u_{2}$ and $u_{2} \wedge u_{0}$.

Note that this is also the case for any other $R^{\prime}=N R$, since $R^{\prime}$ will have the same geometric interpretation as $R$, using a different Euclidean reference.

Using Lemma 5.4 one can recover vectors $u_{i}^{\prime}= \pm u_{i}, i=0,1,2$, up to a common $\operatorname{sign} \epsilon$. With any $u_{3}^{\prime}$ independent from $\left\{u_{0}^{\prime}, u_{1}^{\prime}, u_{2}^{\prime}\right\}$ we will have a Euclidean basis, since it differs from the basis $\left\{u_{0}, u_{1}, u_{2}, u_{3}\right\}$ just by a translation and a dilation.

\section{REFERENCES}

[1] E. Bayro-Corrochano, B. Rosenhahn, A geometric approach for the analysis and computation of the intrinsic parameters, Pattern Recognition 35, pp. 169-186. 2002.

[2] M. Barnabei, A. Brini and G.C. Rota, On the Exterior Calculus of Invariant Theory, Journal of Algebra 96, pp. 120-160. 1985.

[3] N. Bourbaki, Éléments de mathématique. Algèbre. Chapitres 1 à 3, Paris, Hermann, 1970.

[4] S. Carlsson, The Double Algebra: An Effective Tool for Computing Invariants in Computer Vision, S. Carlsson, pp. 145-164 Applications of Invariance in Computer Vision: Joint European- US Workshop, Azores, Portugal, Oct. 1993

[5] D.S. Dummit and R.M. Foote, Abstract algebra. Wiley, 1999, NJ, USA.

[6] O. Faugeras and Q.T. Luong, The Geometry of Multiple Images, The MIT Press, Cambridge, Massachusetts, 2001

[7] P. Gill, W. Murray and M. Wright. Practical Optimization. Academic Press, 1981.

[8] J. Harris, Algebraic Geometry. A first course. Graduate Texts in Mathematics 133, SpringerVerlag, New York, 1992.

[9] R.I. Hartley and A. Zisserman, Multiple View Geometry in Computer Vision. Cambridge University Press, Cambridge, UK, 2000.

[10] A. Heyden, A Common Framework for Multiple-View Tensors European Conference on Computer Vision, Freiburg, Germany, 1998.

[11] A. Heyden and K. Åström, Minimal conditions on intrinsic parameters for Euclidean reconstruction. Asian Conference on Computer Vision, Hong Kong, 1998.

[12] J.Y. Kaminski, M. Fryers, A. Shashua, M. Teicher, Multiple view geometry of non-planar algebraic curves Computer Vision, 2001. ICCV 2001 (2), pp. 181-186.

[13] S.J. Maybank and O. Faugeras, A Theory of Self-Calibration of a Moving Camera. International Journal of Computer Vision 8, pp. 123-152. 1992.

[14] M. Pollefeys, R. Koch and L. Van Gool, Self-Calibration and Metric Reconstruction in spite of Varying and Unknown Internal Camera Parameters, Proc. ICCV'98.

[15] J. Ponce, On Computing Metric Upgrades of Projective Reconstructions Under the Rectangular Pixel Assumption. Proc. SMILE 2000.

[16] H. Pottmann, J. Wallner, Computational Line Geometry, Springer-Verlag, Germany, 2001.

[17] J.G. Semple and G.T. Kneebone, Algebraic Projective Geometry, Oxford University Press, 1998.

[18] Y. Seo and A. Heyden, Autocalibration from the orthogonality constraints, ICCV'00.

[19] W. Triggs, Matching Constraints and the Joint Image. IEEE Int. Conf. Computer Vision, Cambridge, MA, June 1995.

[20] W. Triggs, Auto-calibration and the absolute quadric. Proc. IEEE Conference on Computer Vision and Pattern Recognition, pages 609-614. 1997.

[21] A. Valdés and J.I. Ronda, Camera autocalibration and the calibration pencil. Journal of Mathematical Imaging and Vision 23, pp. 167-174, 2005.

[22] R.O. Wells, Differential Analysis on Complex Manifolds, Springer-Verlag, New York, 1980. 